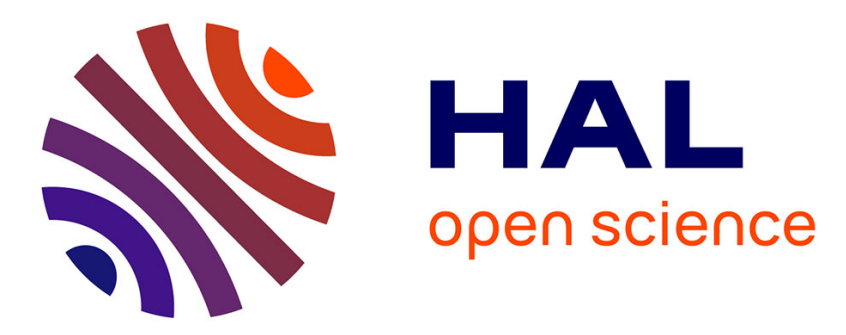

\title{
Uncertainty quantification for multidisciplinary launch vehicle design using model order reduction and spectral methods
}

Loic Brevault, Mathieu Balesdent

\section{- To cite this version:}

Loic Brevault, Mathieu Balesdent. Uncertainty quantification for multidisciplinary launch vehicle design using model order reduction and spectral methods. Acta Astronautica, 2021, 187, pp.295-314. hal-03523336

\section{HAL Id: hal-03523336 \\ https://hal.science/hal-03523336}

Submitted on 12 Jan 2022

HAL is a multi-disciplinary open access archive for the deposit and dissemination of scientific research documents, whether they are published or not. The documents may come from teaching and research institutions in France or abroad, or from public or private research centers.
L'archive ouverte pluridisciplinaire $\mathbf{H A L}$, est destinée au dépôt et à la diffusion de documents scientifiques de niveau recherche, publiés ou non, émanant des établissements d'enseignement et de recherche français ou étrangers, des laboratoires publics ou privés. 


\title{
Uncertainty quantification for multidisciplinary launch vehicle design using model order reduction and spectral methods
}

\author{
Loic Brevault ${ }^{a, *}$, Mathieu Balesdent ${ }^{a}$ \\ ${ }^{a}$ ONERA DTIS, Université Paris Saclay, F-91123 Palaiseau Cedex, France
}

\section{ARTICLE INFO}

\section{Keywords:}

Uncertainty quantification

Launch vehicle design

Optimal control

Karhunen-Loève

Polynomial Chaos Expansion

\begin{abstract}
A B S T R ACT
The early design phase of launch vehicles often involves low fidelity models that are characterized by a high level of modeling uncertainties. These uncertainties have to be propagated into the whole design process in order to ensure the robustness of the found vehicle architecture. Launch vehicle design involves trajectory optimization that induces a large computational cost for the uncertainty propagation phase using nested loop approach (outer uncertainty loop and inner optimal control loop). In this paper, a methodology is proposed in order to build a surrogate model of the uncertainty propagation phase on the trajectory optimization in order to carry out the uncertainty quantification at a reduced cost. The proposed approach couples reduced order model and spectral methods in order to allow to generate optimal launch vehicle trajectories as functions of the input uncertainties. The method is applied to two-stage-to-orbit launch vehicle design in several uncertainty quantification analyses (reliability analysis, sensitivity analysis, quantile estimation).
\end{abstract}

\section{Introduction}

The design of a new launch vehicle is a process that is in general decomposed into different successive phases: the early design phase, followed by the preliminary phase, the detailed phase, up to the manufacturing and its operational life. In the early design phase, various launch vehicle architectures, technologies and life cycle options are assessed in order to identify the most suited launcher for a dedicated market. Therefore, the associated design space in the early design phase is large as only few characteristics are frozen. Designing a launch vehicle involves a multidisciplinary process which couples disciplines such as propulsion, aerodynamics, structure or trajectory. The launch vehicle performance assessment requires a coupled analysis between the disciplines called MultiDisciplinary Analysis (MDA) [3] which consists in solving a nonlinear system of equations to satisfy the interdisciplinary couplings. Due to the large design space that has to be explored, in the early design phase, low fidelity models are commonly used to evaluate the launch vehicle performance through a MDA. These types of disciplinary models allow a limited computational cost associated to the repeated evaluations of the disciplines for instance in a Multidisciplinary Design Optimization (MDO) framework. However, due to physical simplifications (e.g., Euler-Bernoulli beam theory instead of Timoshenko beam theory), numerical relaxation (e.g., convergence criterion, coarse mesh), compared to higher fidelity models, the predicted performance of the launch vehicle is uncertain. Therefore, it is essential to account for the modeling uncertainty into the early design phase of a launch vehicle. In practice, uncertain variables are often described according to the probability formalism and sampling-based techniques (such as Crude Monte Carlo) are used for uncertainty quantification [8]. However, including uncertainty quantification into the assessment of launch vehicle performance is a challenge as it combines the computational cost of MDA, uncertainty propagation and optimization. In addition, for launch vehicle design, the trajectory module is a key discipline in the MDA. It involves a dynamical system including control variables for the launch vehicle. The dynamical system is expressed through a system of equations of motion describing the dynamics of the state variables (e.g., altitude, velocity, flight path angle) under a certain control law (defined by the control variables). In general, the trajectory discipline includes an auxiliary optimization problem to find the optimal trajectory control variables for the considered launcher at each iteration step of the MDO process. Therefore, the propagation of modeling uncertainty (coming from the different disciplines) to assess the launch vehicle performance requires to repeat trajectory optimization problem solving which is computationally intensive.

\footnotetext{
*Corresponding author

loic.brevault@onera.fr (L. Brevault); mathieu.balesdent@onera.fr (M. Balesdent) $\operatorname{ORCID}(\mathrm{s})$ :
} 
Nested approach

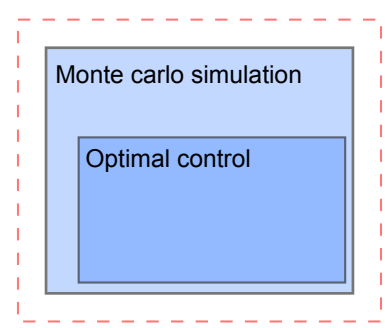

Expanded approach

Expanded optimal control with polynomial chaos

Figure 1: Existing approaches for uncertainty quantification with optimal control trajectory optimization

In the literature, several approaches have been proposed to propagate uncertainty through the MDA for the assessment of the performance of a launch vehicle. Two main approaches exist in the literature to propagate uncertainty in the multidisciplinary problem (Figure 1). The first one $[8,11,39]$ is a nested loop method with Monte Carlo Simulation (MCS) at the outer level and optimal control at the inner level. Therefore, for each realization of the uncertain variables, an optimal control problem is solved resulting in a high computational cost. This approach offers the possibility to get exact state variable realizations (e.g., altitude, velocity) with an optimal trajectory for each realization of the uncertain modeling variables. However, in this case, the calculation cost combines the cost of uncertainty propagation, the one of MDA performed for each sample of the uncertainty propagation, and the trajectory optimization which has to be carried out at each loop induced by the MDA. Consequently, a large number of the trajectory simulations has to be performed (see Figure 2) and only a limited number of samples may be propagated. For example, if the trajectory optimal control problem calls in average 200 trajectory simulations, MDA requires 5 loops between the different disciplines and 10000 samples are used for uncertainty propagation, the trajectory has to be simulated $10^{7}$ times which is not achievable.

The second approach [16, 24, 36, 38] (for instance applied to aircraft trajectories or entry descent and landing trajectories) consists in decomposing the optimal control problem with spectral methods (e.g., Polynomial Chaos Expansion - PCE) and to solve a single optimization problem over an extended design space. It allows to transform the nested loop approach into a single deterministic optimization problem by a decomposition of the state variables according to PCE. However, in practice, the resulting optimization problem is very difficult to solve (due to the high dimension of the search space) and computationally expensive. As mentioned in [36], this type of approach is in practice applied to problems with a limited number of uncertain variables $(\leq 3)[16,24,38]$.

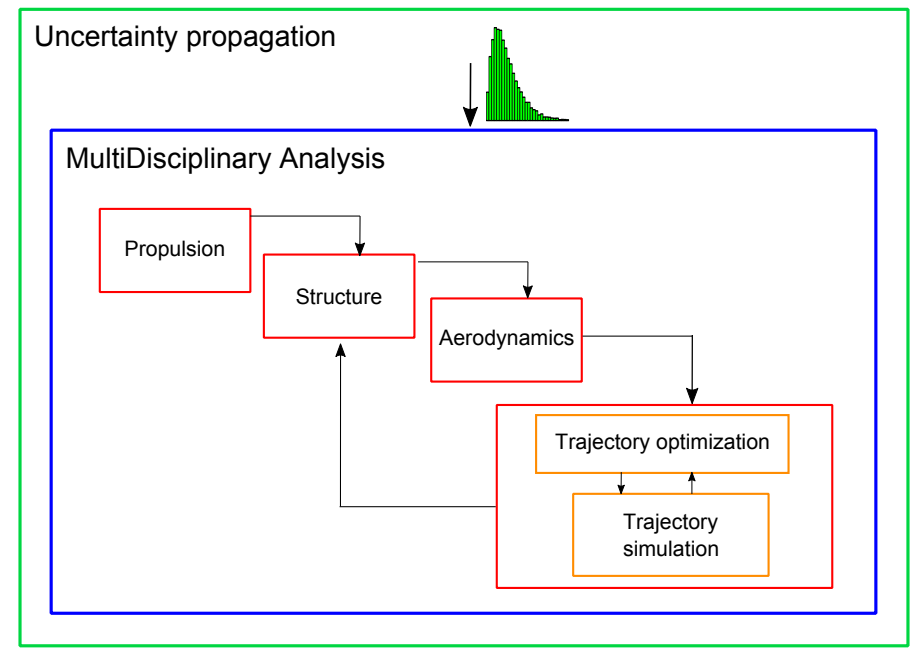

Figure 2: Involved loops for uncertainty propagation inducing MDA and trajectory optimization

The main objective of this paper is to derive an uncertainty propagation technique for launch vehicle design with 
a reasonable computational cost. It is based on surrogate model allowing to handle the numerical cost of uncertainty quantification for coupled problems involving trajectory optimization. The proposed approach couples model order reduction and spectral methods to define a surrogate model that may be used in different uncertainty quantification studies about the performance of the launch vehicle (e.g., statistical moment estimation of the state variables, reliability analysis, sensitivity analysis). The rest of the paper is organized as follows. In Section 2, the proposed methodology is described. Starting from the optimal control problem formulation, the different steps of the proposed approach are then presented. In Section 3, this approach is applied to the optimal control problem of a two-stage-to-orbit expendable launch vehicle. Different uncertainty quantification analyses are performed: computation of statistical moment of time dependent state variables, sensitivity analysis and reliability analysis. The proposed approach is compared to the nested loop method both in terms of resulting accuracy and computational cost.

\section{Model order reduction and spectral methods for launch vehicle trajectory under uncertainty}

\subsection{Optimal control problem formulation}

Given architectural design variables $\mathbf{z} \in \mathbb{R}^{d}$ (e.g., stage diameters, chamber pressures, nozzle geometry shape), the multidisciplinary analysis involves in general an optimal control problem within the trajectory. Indeed, the trajectory module may be considered as an analysis discipline or a simulation discipline depending on the handling of the trajectory control variables [3]. If the control variables $\mathbf{w}$ for the launch vehicle are handled at the system level, the trajectory discipline is only a simulation discipline propagating the system dynamic using the Ordinary Differential Equations (ODEs). However, if the control variables are handled at the discipline level, the trajectory discipline is an analysis discipline and an optimal control problem is solved to determine the best value of the control variables considering an objective function while satisfying path constraints. In the following, an analysis discipline is considered for the trajectory discipline. The corresponding optimal control problem for the trajectory discipline may be formulated as follows:

$$
\begin{aligned}
\min & J(\mathbf{z}, \mathbf{y}, \mathbf{x}(t), \mathbf{w}(t), \mathbf{U}) \\
\text { w.r.t. } & \mathbf{w}(t) \\
\text { s.t. } & \dot{\mathbf{x}}(t)=f(\mathbf{z}, \mathbf{y}, \mathbf{x}(t), \mathbf{w}(t), \mathbf{U}) \\
& \mathbf{g}(\mathbf{z}, \mathbf{y}, \mathbf{x}(t), \mathbf{w}(t), \mathbf{U}) \leq 0 \\
& \mathbf{x}_{\min } \leq \mathbf{x}(t) \leq \mathbf{x}_{\max } \quad \forall t \in \mathcal{T} \\
& \mathbf{w}_{\min } \leq \mathbf{w}(t) \leq \mathbf{w}_{\max } \quad \forall t \in \mathcal{T} \\
& \mathbf{x}\left(t_{0}\right)=\mathbf{x}_{0} \\
& \mathbf{x}\left(t_{f}\right)=\mathbf{x}_{f}
\end{aligned}
$$

where $\mathbf{z} \in \mathbb{R}^{d}$ is the vector of architectural design variables, $\mathbf{y} \in \mathbb{R}^{s}$ is the vector of input coupling variables to the trajectory, $\mathbf{x}(t)$ is the time dependant state variable vector $(t \in \mathcal{T})$ with $t_{0}$ and $t_{f}$ the initial and final time instants. Moreover, $\mathbf{w}(t)$ is the control variable vector (for example the pitch angle profile as a function of time). The state variable vector follows a system of dynamic equations $\dot{\mathbf{x}}(t)=f(\mathbf{z}, \mathbf{y}, \mathbf{x}(t), \mathbf{w}(t), \mathbf{U})$ with $\mathbf{U} \in \mathbb{R}^{p}$ is considered as a constant vector for the moment and will be used to represent the uncertainties later. $\mathbf{g}(\mathbf{z}, \mathbf{y}, \mathbf{x}(t), \mathbf{w}(t), \mathbf{U}) \leq 0$ is the vector of path constraints. The state and control variable vectors are defined within an interval with corresponding lower and upper bounds. $J(\cdot)$ is the performance criterion for the optimal control problem (e.g., overall consumption during the trajectory). Two main categories of optimization techniques may be distinguished to solve such an optimization trajectory problem [5]. These are the direct and the indirect methods. The direct methods are often used as they are easy to implement and can be relatively well initialized comparatively to the indirect methods. In this kind of approaches, the control vector $\mathbf{w}(t, \boldsymbol{\omega})$ is defined by a finite set of parameters $\omega$ which are controlled by the optimizer in order to satisfy the optimality conditions. This results in a Non-Linear Programming problem (NLP) which may be solved using classical gradient-based optimization algorithms [6] or alternatively by metaheuristic algorithms [18]. For more details about optimal control techniques for launch vehicle trajectory, please refer to [5, 28].

In the presence of uncertainty, $\mathbf{U}$ is not considered as a constant vector but as an aleatory vector defined over a probability space $(\Upsilon, \mathcal{A}, \mathbb{P})$ with $\Upsilon$ the universal set, $\mathcal{A}$ a $\sigma$-algebra and the probability measure $\mathbb{P}$. $\mathbf{U}$ is defined using a joint probability density function $\phi_{\mathbf{U}}(\cdot)$ on its definition domain $\Omega$. Due to the presence of uncertain variables $\mathbf{U}$, the 
state variable vector become stochastic process given a control profile. It is noted $\mathbf{X}: \mathcal{T} \times \Omega \rightarrow \mathbb{R}^{q}$ corresponding a multivariate stochastic process of the state variables of dimension $q$ with $\mathcal{T}$ the time domain and $\mathbf{X}(t, \mathbf{U}) \in \mathbb{R}^{q}$. To introduce some notations associated to the stochastic process, $\mathbf{X}_{t}: \Omega \rightarrow \mathbb{R}^{q}$ is the random variable vector at time $t \in \mathcal{T}$ defined by $\mathbf{X}_{t}(\mathbf{u})=\mathbf{X}(t, \mathbf{u})$ and $\mathbf{X}(\mathbf{u}): \mathcal{T} \rightarrow \mathbb{R}^{q}$ is a realization of the process for a given realization of $\mathbf{U}$ defined by $\mathbf{X}(\mathbf{u})(t)=\mathbf{X}(t, \mathbf{u})$. The autocovariance of the stochastic process for two different times $t_{1}, t_{2} \in \mathcal{T}^{2}$ is defined by $C_{\mathbf{X X}}\left(t_{1}, t_{2}\right)=\operatorname{cov}\left[\mathbf{X}_{t 1}, \mathbf{X}_{t 2}\right]=\mathbb{E}\left[\left(\mathbf{X}_{t 1}-\boldsymbol{\mu}_{t 1}\right)\left(\mathbf{X}_{t 2}-\boldsymbol{\mu}_{t 2}\right)\right]$ with $\boldsymbol{\mu}_{t i}$ the mean associated to the stochastic process $\boldsymbol{\mu}_{t}=\mathbb{E}\left[\mathbf{X}_{t}\right]$.

Therefore it is possible to define the following application of interest:

$$
\mathbf{U} \sim \phi_{\mathbf{U}} \stackrel{\mathcal{Q}_{\mathbf{X}^{*}}}{\longrightarrow} \mathbf{X}^{*}(t, \mathbf{U})
$$

with $\mathbf{X}^{*}(t, \mathbf{U})$ the resulting optimal stochastic process trajectories for the state variables obtained by solving the optimal control problem according to the different realizations of $\mathbf{U}$. In the rest of the paper, for notation simplicity, the derivations are made for one component of the stochastic process output.

\section{Proposed approach}

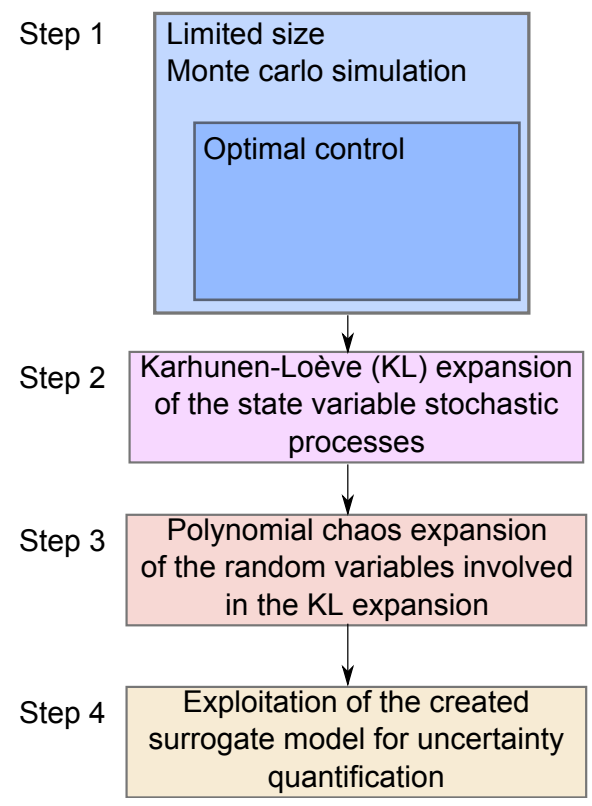

Figure 3: Proposed strategy for uncertainty quantification with optimal control trajectory optimization

Using $\mathcal{Q}_{X^{*}}(\cdot)$, it is possible to perform uncertainty quantification of different quantities of interest involving the optimal stochastic process for the state variables (or control variables). For instance, it is possible to estimate the mean function of the state variables (of the altitude, the velocity, the dynamic pressure, etc.) or to estimate quantiles over the state variables to define a flight envelope considering the uncertain input vector $\mathbf{U}$. It is also possible to define a probability of exceedance for a state variable to be above a certain threshold (e.g., probability that the dynamic pressure is above a threshold). Eventually, it could be interesting to determine along the trajectory a sensitivity index of the state variables with respect to the input uncertain variables. However, all these uncertainty quantification estimations require repeated evaluations of the application $\mathcal{Q}_{X^{*}}(\cdot)$ which is computationally prohibitive as it involves the solving of an optimal control problem. As discussed in the previous section, the solving of optimal control problem under uncertainty for launch vehicle trajectory is challenging. In order to limit the computational cost for uncertainty quantification, the proposed approach relies on the definition of a surrogate model $\hat{\mathcal{Q}}_{X^{*}}(\cdot)$ of the application $\mathcal{Q}_{X^{*}}(\cdot)$ relying on model order reduction [17, 21, 35] and Polynomial Chaos Expansion [2, 15, 33, 37]. These techniques (Karhunen-Loève decomposition and Polynomial Chaos Expansion) are classically used within the context of uncertainty quantification when stochastic processes are involved as they have been developed for the purpose of uncertainty propagation. In the following sections, the different steps of the proposed strategy are detailed (Figure 3). 


\subsection{Step 1: Limited size Monte Carlo Simulation}

In a first step, a Monte Carlo Simulation (MCS) of $M$ samples is carried out. It consists in generating samples of the input vector $V_{M}=\left[\mathbf{u}_{1}, \ldots, \mathbf{u}_{M}\right]$ by sampling according to the joint Probability Density Function (PDF) $\phi_{\mathbf{U}}(\cdot)$. For each sample $\mathbf{u}_{k \in[1, \ldots, M]}$ of $\mathcal{V}_{M}$, a deterministic optimal control problem is solved in order to determine the resulting realization of the optimal stochastic processes $\mathbf{x}^{*}\left(t, \mathbf{u}_{k}\right)$ and $\mathbf{w}^{*}\left(t, \mathbf{u}_{k}\right)$. In practice, as the resulting stochastic process realization is defined by the solving of an optimization problem involving an ODE, the stochastic process realization is discretized over a mesh in time $\mathcal{T}_{N}$ such that $\left[\mathbf{x}\left(t_{1}, \mathbf{u}_{k}\right), \cdots, \mathbf{x}\left(t_{N}, \mathbf{u}_{k}\right)\right]$ with $N$ vertices. Once the $M$ deterministic optimal control problems have been solved, it results $M$ realizations (also called sample paths) of the optimal stochastic processes for the state variables and the control variables. Because of the need of accuracy in the integration of ODE, the number of vertices $N$ in each realization of the stochastic process is in general substantial. Therefore, the discretized stochastic process consists of $N$ time-correlated random variables. In the step 2, using the $M$ realizations of the stochastic process $\mathbf{X}^{*}(t, \mathbf{U})$, this latter is decomposed according to Karhunen-Loève expansion [17, 21, 35].

\subsection{Step 2: Karhunen-Loève expansion}

In order to be able to construct a surrogate model of the application $\mathcal{Q}_{X^{*}}(\cdot)$, due to the high dimension of the output stochastic process, a proper decomposition is required. The Karhunen-Loève (KL) theorem [17, 21, 35] states that a square integrable stochastic process can be represented by a linear combination of orthogonal functions, typically taken to be eigenfunctions of the autocovariance function of the stochastic process, with random coefficients. The KL expansion is based on the spectral decomposition of its autocovariance function. The set of deterministic functions over which any realization of the stochastic process is expanded is defined by an eigenvalue problem. The eigenvalues and eigenfunctions are determined by solving the second kind Fredholm equation associated to the autocovariance function $C_{\mathbf{X}^{*} \mathbf{X}^{*}}(\cdot, \cdot)$ of the stochastic process (noted $C(\cdot, \cdot)$ for simplicity):

$$
\int_{\mathcal{T}} C(s, t) \mathcal{L}_{k}(t) \mathrm{d} t=\lambda_{k} \mathcal{L}_{k}(s) \quad \forall s \in \mathcal{T}
$$

where $\left(\lambda_{k}\right)_{k \geq 1}$ are the eigenvalues and $\left(\mathcal{L}_{k}\right)_{k \geq 1}$ the associated sequence of eigenfunctions. $\left(\mathcal{L}_{k}\right)_{k \geq 1}$ form a complete orthogonal basis of $L^{2}(\mathcal{T})$. It results that any realization of the stochastic process may be expanded over this basis:

$$
\mathbf{X}^{*}(t, \mathbf{u})=\mu(t)+\sum_{k=1}^{\infty} \sqrt{\lambda_{k}} \xi_{k}(\mathbf{u}) \mathcal{L}_{k}(t)
$$

where $\left(\xi_{k}\right)(\mathbf{u})_{k \geq 1}$ corresponds to the coordinates of the realization of the stochastic process with respect to the deterministic function $\mathcal{L}_{k}(\cdot)$. Moreover, considering all possible realizations of the stochastic process, $\left(\xi_{k}\right)_{k \geq 1}$ forms a set of orthonormal random variables. Due to the orthonormality of the eigenfunctions, each random variable $\xi_{k}(\mathbf{u})$ is determined by a linear transform:

$$
\xi_{k}(\mathbf{u})=\frac{1}{\sqrt{\lambda_{k}}} \int_{\mathcal{T}}\left(\mathbf{X}^{*}(t, \mathbf{u})-\mu(t)\right) \mathcal{L}_{k}(t) \mathrm{d} t
$$

In practice, the KL decomposition is truncated and limited to the most first $N_{k}$ significant modes (the most significant eigenvalues): $\mathbf{X}^{*}(t, \mathbf{u}) \simeq \mu(t)+\sum_{k=1}^{N_{k}} \sqrt{\lambda_{k}} \xi_{k}(\mathbf{u}) \mathcal{L}_{k}(t)$. The eigenfunction basis $\mathcal{L}_{k}(\cdot)$ is optimal in the sense that the mean square error (integrated over $\Omega$ ) resulting from a truncation after the first $N_{k}$ terms is minimal compared to any alternative $L^{2}(\mathcal{T})$ basis.

In the present case, the covariance function associated to the optimal stochastic process $\mathbf{X}^{*}$ is not explicitly known but only accessible through the $M$ realizations $\left(\mathbf{X}_{1}^{*}, \cdots, \mathbf{X}_{M}^{*}\right)$ obtained during the Step 1. Therefore, for a centered stochastic process, $C=\frac{1}{M} \tilde{X} \tilde{X}^{T}$ with $\tilde{X}=\left(\mathbf{X}_{1}^{*}|\cdots| \mathbf{X}_{M}^{*}\right)$ the snapshot matrix constituted of the $M$ realizations of the stochastic process.

The determination of the eigenvalues and eigenfunctions require to solve the second kind Fredholm equation numerically using for instance a Galerkin-type approach [17, 21, 35]. Let consider a basis $h_{i}(\cdot)$ of the Hilbert space $L^{2}(\mathcal{T})$. Each eigenfunction $\mathcal{L}_{k}(\cdot)$ may be represented in this basis by:

$$
\mathcal{L}_{k}(t)=\sum_{i=1}^{N_{k}} d_{i}^{k} h_{i}(t)
$$


where $d_{i}^{k}$ are unknown coefficients. The purpose of the Galerkin approach is to determine the best approximation of $\mathcal{L}_{k}(t)$ considering the truncation. For that, it is possible to define an error function $\epsilon_{N_{k}}(\cdot)$ using Eq.(9) to determine the residual due to the truncation:

$$
\epsilon_{N_{k}}(t)=\sum_{i=1}^{N_{k}} d_{i}^{k}\left[\int_{\mathcal{T}} C\left(t, t^{\prime}\right) h_{i}\left(t^{\prime}\right) \mathrm{d} t^{\prime}-\lambda_{k} h_{i}(t)\right]
$$

By imposing that the residual is orthogonal to the approximating basis, it yields:

$$
\int_{\mathcal{T}} \epsilon_{N_{k}}(t) h_{j}(t) \mathrm{d} t=0 \quad \forall j=1, \ldots, N_{k}
$$

By injecting the expression of the residuals into Eq.(14), the integral rewrites:

$$
\sum_{i=1}^{N_{k}} d_{i}^{k}\left[\int_{\mathcal{T}}\left[\int_{\mathcal{T}} C\left(t, t^{\prime}\right) h_{i}\left(t^{\prime}\right) \mathrm{d} t^{\prime}\right] h_{j}(t) \mathrm{d} t-\lambda_{k} \int_{\mathcal{T}} h_{i}(t) h_{j}(t) \mathrm{d} t\right]=0
$$

This equation may be rewrite with a matrix form:

$$
\mathrm{CD}=\Lambda \mathrm{BD}
$$

with:

$$
\begin{aligned}
\mathbf{C}_{i j} & =\int_{\mathcal{T}} \int_{\mathcal{T}} C\left(t, t^{\prime}\right) h_{i}(t) h_{j}\left(t^{\prime}\right) \mathrm{d} t \mathrm{~d} t^{\prime} \\
\mathbf{B}_{i j} & =\int_{\mathcal{T}} h_{i}(t) h_{j}(t) \mathrm{d} t \\
\mathbf{D}_{i j} & =d_{i}^{k} \\
\Lambda_{i j} & =\delta_{i j} \lambda_{i}
\end{aligned}
$$

where $\delta_{i j}$ is the Kronecker symbol. Equation (16) is a generalized eigenvalue problem [17, 35]. It is solved for eigenvectors $\mathbf{D}$ and the eigenvalues $\boldsymbol{\Lambda}$. In practice, to compute the integrals involved in the matrices $\mathbf{C}$ and $\mathbf{D}$, a quadrature approach is used with the vertices of the mesh $\mathcal{T}_{N}$ used as the quadrature nodes. Moreover, a Singular Value Decomposition is used to simplify the solving of the Eq.(16) [21].

The obtained KL expansion of the output optimal stochastic process decouples the uncertainty and the time dependencies. It is therefore possible to build a surrogate model of $\mathcal{Q}_{X^{*}}(\cdot)$ based on Polynomial Chaos Expansion (PCE) particularly dedicated to uncertainty quantification studies.

\subsection{Step 3: Polynomial Chaos Expansion}

Considering the KL expansion of Step 2, constructed from the available stochastic process realizations obtained at the Step 1, it is possible to create a surrogate model of the application $\mathcal{Q}_{X^{*}}(\cdot)$. In the expansion:

$$
\hat{\mathbf{X}}^{*}(t, \mathbf{u})=\hat{\mu}(t)+\sum_{k=1}^{N_{k}} \sqrt{\hat{\lambda}_{k}} \xi_{k}(\mathbf{u}) \hat{\mathcal{L}}_{k}(t)
$$

$\left(\xi_{k}\right)_{k \geq 1}(\cdot)$ correspond to the uncertainty parts of the decomposition. Therefore, for each random variable associated to the expansion, it is possible to create a PCE $[2,15,33,37]$ of the following application:

$$
\mathbf{u} \sim \phi_{\mathbf{U}} \stackrel{\xi_{k}}{\longrightarrow} \xi_{k}(\mathbf{u})
$$

PCE provides a polynomial approximation of the function $\xi_{k}(\cdot)$ decomposed over an orthogonal basis of polynomials. Considering a vector of $p$ uncertain variables $\mathbf{U}=\left[U_{1}, \ldots, U_{p}\right]$. For each component $i$ of the vector, $\phi_{U_{i}}(\cdot)$ is 
the marginal distribution of the variable $U_{i}$. It is possible to define a family of orthogonal polynomials $\left\{P_{k}^{(i)}, k \in \mathbb{N}\right\}$ such that:

$$
\left\langle P_{j}^{(i)}, P_{k}^{(i)}\right\rangle_{i} \stackrel{\operatorname{def}}{=} \mathbb{E}\left[P_{j}^{(i)}\left(U_{i}\right), P_{k}^{(i)}\left(U_{i}\right)\right]=\int_{\Omega_{i}} P_{j}^{(i)}(u) P_{k}^{(i)}(u) \phi_{U_{i}}(u) \mathrm{d} u=a_{j}^{(i)} \delta_{j k}
$$

where $k$ is the degree of the polynomial $P_{k}^{(i)}$ and $a_{j}^{(i)}$ is the squared norm of $P_{j}^{(i)}$ :

$$
a_{j}^{(i)} \stackrel{\text { def }}{=}\left\|P_{j}^{(i)}\right\|_{i}^{2}=\left\langle P_{j}^{(i)}, P_{j}^{(i)}\right\rangle_{i}
$$

If $U_{i}$ is defined through classical PDF, the associated family of orthogonal polynomials is known [2, 15]. For instance for uniformly distributed variables, the resulting orthogonal polynomial family is the Legendre polynomials. Similarly, for normally distributed variables, the corresponding family is the Hermite polynomials. To define an orthonormal family $\mathcal{F}$, a normalization is defined:

$$
\mathcal{F}_{j}^{(i)}=\frac{P_{j}^{(i)}}{\sqrt{a_{j}^{(i)}}}, \quad i=1, \ldots, p, \quad j \in \mathbb{N}
$$

To build a multivariate orthonormal polynomial basis from the orthonormal univariate family $\left\{\mathcal{F}_{j}^{(i)}\right\}_{j=0}^{\infty}$, a tensor product approach is used:

$$
\mathbf{F}_{\boldsymbol{\alpha}}(\mathbf{u})=\prod_{i=1}^{p} \mathcal{F}_{\alpha_{i}}^{(i)}\left(u_{i}\right)
$$

with $\boldsymbol{\alpha}=\left(\alpha_{1}, \ldots, \alpha_{p}\right) \in \mathbb{N}^{p}$ a multi-index. The total degree of the multivariate polynomial $\mathbf{F}_{\boldsymbol{\alpha}}$ is given by: $|\boldsymbol{\alpha}|=$ $\sum_{i=1}^{p} \alpha_{i}$

PCE allows to decompose $\xi_{k}(\cdot)$ over the multivariate orthonormal polynomial basis such that:

$$
\xi_{k}(\mathbf{u})=\sum_{\alpha \in \mathbb{N}^{p}} \gamma_{\alpha_{k}} \mathbf{F}_{\alpha_{k}}(\mathbf{u})
$$

In practice, the expansion is often truncated using the standard truncation scheme consisting in selecting all the polynomials such that $\left|\boldsymbol{\alpha}_{\boldsymbol{k}}\right|$ is smaller or equal to a given $\zeta$. The multi-indices belong to $\mathcal{A}^{\zeta}=\left\{\boldsymbol{\alpha}_{\boldsymbol{k}} \in \mathbb{N}^{d},\left|\boldsymbol{\alpha}_{\boldsymbol{k}}\right| \leq \zeta\right\}$.

The truncated PCE rewrites:

$$
\hat{\xi}_{k}(\mathbf{u})=\sum_{\alpha_{k} \in \mathcal{A}^{\zeta}} \gamma_{\alpha_{k}} \mathbf{F}_{\alpha_{k}}(\mathbf{u})
$$

The computation of the PCE coefficients may be carried out by non-intrusive techniques such as projection, leastsquare or stochastic collocation [15]. In the following, the least-square approach is employed. Following the PCE decomposition, it holds:

$$
\xi_{k}(\mathbf{u})=\sum_{\alpha_{k} \in \mathcal{A}^{\zeta}} \gamma_{\alpha_{k}} \mathbf{F}_{\alpha_{k}}(\mathbf{u})+\epsilon
$$

with $\epsilon$ the residual terms corresponding to all the polynomials whose index $\boldsymbol{\alpha}_{\boldsymbol{k}}$ is not in the truncation set $\mathcal{A}^{\zeta}$. The least-square method consists in finding the PCE coefficients $\boldsymbol{\Gamma}_{\boldsymbol{k}}=\left\{\gamma_{\boldsymbol{\alpha}_{k}, \boldsymbol{\alpha}_{k} \in \mathcal{A}^{\zeta}}\right\}$, using the M samples generated by $\operatorname{MCS} V_{M}=\left[\mathbf{u}_{1}, \ldots, \mathbf{u}_{M}\right]$ and the corresponding values in the $\operatorname{KL}$ expansion $\left[\xi_{k}\left(\mathbf{u}_{1}\right), \ldots, \xi_{k}\left(\mathbf{u}_{M}\right)\right]$ such that:

$$
\hat{\boldsymbol{\Gamma}}_{\boldsymbol{k}}=\underset{\boldsymbol{\Gamma}_{\boldsymbol{k}}}{\operatorname{argmin}} \frac{1}{M} \sum_{i=1}^{M}\left(\xi_{k}\left(\mathbf{u}_{i}\right)-\sum_{\boldsymbol{\alpha}_{k} \in \mathcal{A}^{\zeta}} \gamma_{\boldsymbol{\alpha}_{k}} \mathbf{F}_{\boldsymbol{\alpha}_{k}}\left(\mathbf{u}_{i}\right)\right)^{2}
$$


Classical least-square solvers may be used to determine the optimal PCE coefficients $\hat{\boldsymbol{\Gamma}}_{\boldsymbol{k}}=\left\{\hat{\gamma}_{\boldsymbol{\alpha}_{k}, \boldsymbol{\alpha}_{k} \in \mathcal{A}^{\zeta}}\right\}$. This PCE decomposition is carried out for each $\xi_{k}(\cdot)_{k \geq 1}$ in the KL decomposition.

At the end, the surrogate model of the application $\mathcal{Q}_{X^{*}}(\cdot)$ is defined by:

$$
\hat{\mathbf{X}}^{*}(t, \mathbf{u})=\hat{\mu}(t)+\sum_{k=1}^{N_{k}}\left(\sqrt{\hat{\lambda_{k}}} \sum_{\alpha_{k} \in \mathcal{A}^{\zeta}}\left(\hat{\gamma}_{\boldsymbol{\alpha}_{k}} \mathbf{F}_{\boldsymbol{\alpha}_{k}}(\mathbf{u})\right) \hat{\mathcal{L}}_{k}(t)\right)
$$

This surrogate model may be used to carry out uncertainty quantification on the launch vehicle trajectory while limiting the associated computational cost.

\subsection{Step 4: Exploitation for uncertainty quantification}

\subsubsection{Statistical moment estimates}

Once the surrogate model has been created with the Steps 1 to 3, it is possible to use it to carry out different uncertainty quantification studies. As it relies on KL and PCE, it is possible to use the specificities of KL and PCE for uncertainty analysis. First, the aleatory variables involved in the KL expansion are uncorrelated random variables. Moreover, due to the fact that PCE are decomposed over orthonormal polynomial basis, mean and standard deviation of the PCE output may be directly computed from the PCE coefficients [15]. Considering these elements, the mean value and the variance of the PCE output write:

$$
\mathbb{E}\left[\xi_{k}(\mathbf{u})\right]=\mathbb{E}\left[\sum_{\boldsymbol{\alpha}_{k} \in \mathcal{A}^{\zeta}} \hat{\gamma}_{\boldsymbol{\alpha}_{k}} \mathbf{F}_{\boldsymbol{\alpha}_{k}}(\mathbf{u})\right]=\hat{\gamma}_{0_{k}}
$$

and:

$$
\mathbb{V}\left[\xi_{k}(\mathbf{u})\right]=\mathbb{V}\left[\sum_{\alpha_{k} \in \mathcal{A}^{\zeta}} \hat{\gamma}_{\boldsymbol{\alpha}_{k}} \mathbf{F}_{\boldsymbol{\alpha}_{k}}(\mathbf{u})\right]=\sum_{\substack{\boldsymbol{\alpha}_{k} \in \mathcal{A}^{\zeta} \\ \alpha_{k} \neq 0}} \hat{\gamma}_{\boldsymbol{\alpha}_{k}}^{2}
$$

Using these elements, it is possible to get the first two statistical moments of the stochastic process of the state variables, for the mean field:

$$
\mathbb{E}\left[\hat{\mathbf{X}}^{*}(t, \mathbf{u})\right]=\hat{\mu}(t)+\sum_{k=1}^{N_{k}}\left(\sqrt{\hat{\lambda}_{k}} \hat{\gamma}_{0_{k}} \hat{\mathcal{L}}_{k}(t)\right)
$$

and the variance:

$$
\mathbb{V}\left[\hat{\mathbf{X}}^{*}(t, \mathbf{u})\right]=\sum_{k=1}^{N_{k}}\left(\sqrt{\hat{\lambda}_{k}} \hat{\mathcal{L}}_{k}(t)\right)^{2} \sum_{\substack{\alpha_{k} \in \mathcal{A} \\ \alpha_{k} \neq 0}} \hat{\gamma}_{\boldsymbol{\alpha}_{k}}^{2}
$$

\subsubsection{Quantile estimates}

The estimation of quantile over the state variable stochastic process is interesting to define trajectory envelop considering the input uncertain variables. Considering the aleatory variable $\xi_{k}(\mathbf{u})$ and $\eta \in\left[0 ., 1\right.$.], the $\eta$-quantile $q_{\eta}$ is defined by:

$$
q_{\eta}=\inf _{v \in \mathbb{R}}\left\{\mathbb{P}\left[\xi_{k}(\mathbf{u}) \leq v\right] \geq \eta\right\}=\inf _{v \in \mathbb{R}}\left\{\Psi_{\xi_{k}}(v) \geq \eta\right\}
$$

with $\Psi_{\xi_{k}}(\cdot)$ the $\mathrm{CDF}$ of $\xi_{k}(\mathbf{u})$.

This definition may be extended to the surrogate model of the stochastic process $\hat{\mathbf{X}}^{*}(t, \mathbf{u})$. In practice, $R$ samples of the input vector $V_{R}=\left[\mathbf{u}_{1}, \ldots, \mathbf{u}_{R}\right]$ are generating according to $\phi_{\mathbf{U}}(\cdot)$. Then, using the surrogate model $\hat{\mathbf{X}}^{*}(t, \mathbf{u}), R$ realizations of the stochastic process corresponding to the input samples are generated. Eventually, for each $t \in \mathcal{T}_{N}$, $\hat{\mathbf{X}}_{t}^{*}$ is a random variable and $R$ realizations of this variable are available. Therefore, the $\eta$-quantile $q_{\eta}$ of $\hat{\mathbf{X}}_{t}^{*}$ may be computed using the empirical estimate of the CDF of $\hat{\mathbf{X}}_{t}^{*}$. Following this approach, it is possible to provide $\eta$-quantile estimate of the stochastic process $\hat{\mathbf{X}}^{*}(t, \mathbf{u})$. For instance, it may be used to determine the $5 \%$ or the $95 \%$ quantiles of the altitude as a function of time along the trajectory considering the input uncertain variables. 


\subsubsection{Probability of failure estimates}

Using the surrogate model $\hat{\mathbf{X}}^{*}(t, \mathbf{u})$ of the stochastic process of the state variables, it is possible to carry out reliability analysis by estimating probability of failures. For instance, it is possible to estimate the probability that an event occurs during the trajectory. Indeed, one could be interested to estimate the probability that one state variable during the trajectory goes out of a defined feasible domain (e.g., trajectory envelop). Concerning launch vehicle trajectory, an important aspect is that the launcher has to be visible at each time by a ground station for safety reasons. Therefore, one might be interested to estimate the probability that at one instant during the trajectory, the launch vehicle is not visible by any ground station. The estimation of this probability of failure requires to know the state variables at each instant of the trajectory. Therefore, the probability of failure could be defined as: $\mathbb{P}\left[\exists t \in \mathcal{T}_{N} \mid \mathbf{X}_{t}(\mathbf{u}) \in \mathcal{B}_{t}\right]$ where $\mathcal{B}_{t}$ is the failure domain that depends on time and corresponds to the ensemble of positions in space that are not visible by any ground station.

Using the surrogate model $\hat{\mathbf{X}}^{*}(t, \mathbf{u})$, it is possible to estimate such a probability of failure using for example a Monte Carlo Simulation technique. In practice, $R$ samples of the input vector $V_{R}=\left[\mathbf{u}_{1}, \ldots, \mathbf{u}_{R}\right]$ are drawn according to the joint PDF $\phi_{\mathbf{U}}(\cdot)$. Using the surrogate model $\hat{\mathbf{X}}^{*}(t, \mathbf{u}), R$ realizations of the stochastic process corresponding to the input samples are generated. Then, the probability of failure is computed by MCS:

$$
\hat{\mathbb{P}}=\frac{1}{R} \sum_{i=1}^{R} \mathbf{1}_{\exists t \in \mathcal{T}_{N} \mid \mathbf{X}_{t}(\mathbf{u}) \in \mathcal{B}_{t}}
$$

where $\mathbf{1}_{\exists t \in \mathcal{T}_{N} \mid \mathbf{X}_{t}(\mathbf{u}) \in \mathcal{B}_{t}}$ is equal to 1 if $\exists t \in \mathcal{T}_{N} \mid \mathbf{X}_{t}(\mathbf{u}) \in \mathcal{B}_{t}$ and 0 otherwise.

\subsubsection{Sensitivity analysis with Sobol' technique}

Variance-based Sensitivity Analysis (SA) relies on a functional decomposition of the variance of the output of interest to provide sensitivity indices, that can be interpreted as the proportion of variability of the output that can be apportioned to each input. It decomposes the output variance into contributions of different elements (marginal effects and interactions between the input variables). In the following, the function $Y=\xi(\mathbf{u})$ is considered ( $k$ as been dropped for more clarity in the notations). Let consider that the coordinates of $\mathbf{U}$ are distributed according to a uniform distribution over the unit-hypercube $\mathbb{R}^{p}$.

The Sobol' decomposition is given by [32]:

$$
Y=\xi(\mathbf{U})=\xi_{0}+\sum_{j=1}^{p} \xi_{j}\left(U_{j}\right)+\sum_{i<j}^{p} \xi_{i j}\left(U_{i}, U_{j}\right)+\cdots+\xi_{1 \ldots p}\left(U_{1}, \ldots, U_{p}\right),
$$

where $\xi_{0}=\mathbb{E}[\xi(\mathbf{U})]=\int_{\Omega} \xi(\mathbf{u}) \phi(\mathbf{u})$ du with $\Omega$ the $p$-dimensional cube $[0,1]^{p}$ in which the input variables are distributed according to the uniform $\operatorname{PDF} \phi(\cdot)$. Furthermore,

$$
\begin{aligned}
\xi_{j}\left(U_{j}\right) & =\mathbb{E}\left[\xi(\mathbf{U}) \mid U_{j}\right]-\xi_{0}, \\
\xi_{i j}\left(U_{i}, U_{j}\right) & =\mathbb{E}\left[\xi(\mathbf{U}) \mid U_{i}, U_{j}\right]-\mathbb{E}\left[\xi(\mathbf{U}) \mid U_{i}\right]-\mathbb{E}\left[\xi(\mathbf{U}) \mid U_{j}\right]+\xi_{0},
\end{aligned}
$$

and $\xi_{1, \ldots, p}\left(U_{1}, \ldots, U_{p}\right)$ is determined by the difference between $\xi(\mathbf{U})$ and the sum of all the increasing dimension functions in order for Eq.(37) to be verified. Moreover, $\forall l \in\{1, \ldots, s\} ; \forall\left\{j_{1}, \ldots, j_{s}\right\} \subseteq\{1, \ldots, p\}$

$$
\int_{\Omega} \xi_{j_{1}, \ldots, j_{s}}\left(u_{j_{1}}, \ldots, u_{j_{s}}\right) \mathrm{d} u_{j_{l}}=0
$$

is verified for each function of the decomposition [30]. The orthogonality of the Sobol' decomposition functions may be proved from Eq.(37) [32].

Sobol' derives the Sobol' indices [30] to quantify the partition of the output variance [31] based on the functional decomposition Eq.(37). With the decomposition of the function $\xi(\cdot)$ into the sum of functions of increasing dimensions and by using the decomposition of the variance [32], it follows:

$$
\mathbb{V}[Y]=\sum_{j=1}^{p} \mathbb{V}_{j}[Y]+\sum_{i<j}^{p} \mathbb{V}_{i j}[Y]+\cdots+\mathbb{V}_{123 \ldots p}[Y]
$$


with $\mathbb{V}$ the variance, and $\mathbb{V}_{j}[Y]$ as defined in Eqs.(43-44). The variability of the output $Y$ due to all the input variables except $U_{j}$ is analyzed by fixing the input variable $U_{j}$ at a realization $u_{j}$ :

$$
\mathbb{V}\left[Y \mid U_{j}=u_{j}\right]=\mathbb{E}\left[Y^{2} \mid U_{j}=u_{j}\right]-\mathbb{E}\left[Y \mid U_{j}=u_{j}\right]^{2} .
$$

The expectation of the conditional variance is estimated to account for all the possible $u_{j}$ leading to:

$$
\mathbb{E}\left[\mathbb{V}\left[Y \mid U_{j}=u_{j}\right]\right]=\int_{\Omega_{U_{j}}} \mathbb{V}\left[Y \mid U_{j}=u_{j}\right] \phi_{U_{j}}\left(u_{j}\right) \mathrm{d} u_{j} .
$$

Given the total variance:

$$
\mathbb{V}[Y]=\mathbb{V}\left[\mathbb{E}\left[Y \mid U_{j}\right]\right]+\mathbb{E}\left[\mathbb{V}\left[Y \mid U_{j}\right]\right],
$$

the value $\mathbb{V}\left[\mathbb{E}\left[Y \mid U_{j}\right]\right]$ may be used for SA. It increases as the contribution of the variable $U_{j}$ with respect to the variance of Y increases. To have a normalized quantity, the first order Sobol' index $S_{j}$ for the input variable $U_{j}$ and second order Sobol' index $S_{i j}$ for the interaction between $U_{i}$ and $U_{j}$ are defined such that:

$$
\begin{aligned}
S_{j} & =\frac{\mathbb{V}\left[\mathbb{E}\left[Y \mid U_{j}\right]\right]}{\mathbb{V}[Y]}=\frac{\mathbb{V}_{j}}{\mathbb{V}[Y]}, \\
S_{i j} & =\frac{\mathbb{V}\left[\mathbb{E}\left[Y \mid U_{i}, U_{j}\right]\right]-\mathbb{V}_{i}-\mathbb{V}_{j}}{\mathbb{V}[Y]}=\frac{\mathbb{V}_{i j}}{\mathbb{V}[Y]} .
\end{aligned}
$$

The first order Sobol' index quantifies the part of variance of $Y$ due to $U_{j}$, referred to as main effect. The second order Sobol' index allows at measuring the importance of the interaction between two input variables $U_{i}$ and $U_{j}$. The same principle may be used to derive the Sobol' indices of order 3, 4, etc. The total Sobol' indices $S T_{j}$ are the sum of all the Sobol' indices relative to $U_{j}$

$$
S T_{j}=\sum_{j \sharp i} S_{i},
$$

where $j \sharp i$ stands for all the $S_{i 1, \ldots, i d}$ terms that include the index $j$. For instance, $S T_{1}$ includes $S_{1}, S_{12}, \ldots, S_{1 p}$, $S_{123}, \ldots, S_{123 \ldots p}$. Total Sobol' indices measure the part of the output variance explained by all the effects in which the input variable $j$ plays a part (the first order and all the higher orders).

For black-box functions, the Sobol' indices may not be analytically derived and have to be numerically estimated. Several methods may be employed. Crude Monte Carlo method is traditionally used to estimate Sobol' indices. Other sampling schemes may be performed such as Jansen's [23] or Fourier Amplitude Sensitivity Test (FAST) [29]. The Sobol' index calculations are computationally expensive and require a large number of calls to the studied function. The Sobol' method is applicable to all the cases for which variances are finite (linear or nonlinear, monotonic or non monotonic functions).

As Sobol' decomposition and PCE are sums of orthogonal functions, it is possible to use PCE characteristics to provide the Sobol' indices as a by-product of the surrogate model creation of the state variable stochastic processes [19, 34]. Let us define $\mathcal{A}_{B}=\left\{\boldsymbol{\alpha} \in \mathcal{A}: \alpha_{k} \neq 0\right.$ if and only if $\left.k \in B\right\}$, the set of multi-indices that depend only on a subset of variables $B=\left\{i_{1}, \cdots, i_{s}\right\} \subset\{1, \cdots, p\}$. The union of all these sets are equal to $\mathcal{A}$. Therefore, it is possible to reorganize the PCE decomposition in order to exhibit the Sobol' decomposition (sum of increasing dimension polynomials):

$$
\hat{\xi}(\mathbf{u})=\hat{\gamma}_{0}+\sum_{\substack{B \subset\{1, \cdots, p\} \\ B \neq 0}} K_{B}\left(\mathbf{u}_{B}\right)
$$

with $K_{B}\left(\mathbf{u}_{B}\right)=\sum_{\alpha \in \mathcal{A}_{B}} \hat{\gamma}_{\alpha} \mathbf{F}_{\alpha}(\mathbf{u})$ 
Therefore, in the case $B$ corresponds to the variable $\mathbf{U}_{i}$, because of the orthogonal decomposition:

$$
\mathbb{V}_{i}=\mathbb{V}\left[K_{i}\left(\mathbf{U}_{i}\right)\right]=\sum_{\alpha \in \mathcal{A}_{i}} \hat{\gamma}_{\alpha}^{2}
$$

The Sobol' indices at any order may be estimated by a simple combination of the squares of the PCE coefficients. Therefore, for the first-order Sobol' indices:

$$
\hat{S}_{i}=\frac{\sum_{\alpha \in \mathcal{A}_{i}} \hat{\gamma}_{\boldsymbol{\alpha}}^{2}}{\sum_{\boldsymbol{\alpha} \in \mathcal{A}, \boldsymbol{\alpha} \neq 0} \hat{\gamma}_{\boldsymbol{\alpha}}^{2}}
$$

with $\mathcal{A}_{i}=\left\{\boldsymbol{\alpha} \in \mathcal{A}: \alpha_{i}>0, \alpha_{j \neq i}=0\right\}$. Similarly, the total order Sobol' indices are given by:

$$
\widehat{S T}_{i}=\frac{\sum_{\alpha \in \mathcal{A}_{i}^{T}} \hat{\gamma}_{\alpha}^{2}}{\sum_{\alpha \in \mathcal{A}, \alpha \neq 0} \hat{\gamma}_{\alpha}^{2}}
$$

with $\mathcal{A}_{i}^{T}=\left\{\alpha \in \mathcal{A}: \alpha_{i}>0\right\}$.

Considering all these elements, it is possible to derive the first order and total order Sobol' indices for the stochastic process of the state variables:

$$
\hat{S}_{\hat{\mathbf{X}}^{*}\left(t, u_{i}\right)}=\frac{\sum_{k=1}^{N_{k}}\left(\sqrt{\hat{\lambda_{k}}} \hat{\mathcal{L}}_{k}(t)\right)^{2} \sum_{\alpha_{k} \in \mathcal{A}_{i}^{\zeta}} \hat{\gamma}_{\alpha_{k}}^{2}}{\sum_{k=1}^{N_{k}}\left(\sqrt{\hat{\lambda}_{k}} \hat{\mathcal{L}}_{k}(t)\right)^{2} \sum_{\substack{\alpha_{k} \in \mathcal{A} \zeta \\ \alpha_{k} \neq 0}} \hat{\gamma}_{\alpha_{k}}^{2}}
$$

and:

$$
\widehat{S T}_{\hat{\mathbf{X}}^{*}\left(t, u_{i}\right)}=\frac{\sum_{k=1}^{N_{k}}\left(\sqrt{\hat{\lambda}_{k}} \hat{\mathcal{L}}_{k}(t)\right)^{2} \sum_{\boldsymbol{\alpha}_{k} \in \mathcal{A}_{i}^{T \zeta}} \hat{\gamma}_{\boldsymbol{\alpha}_{k}}^{2}}{\sum_{k=1}^{N_{k}}\left(\sqrt{\hat{\lambda}_{k}} \hat{\mathcal{L}}_{k}(t)\right)^{2} \sum_{\substack{\boldsymbol{\alpha}_{k} \in \mathcal{A} \zeta \\ \alpha_{k} \neq 0}} \hat{\gamma}_{\boldsymbol{\alpha}_{k}}^{2}}
$$

The proposed approach presented in Section 2 allows to construct a surrogate model dedicated to uncertainty propagation for launch vehicle optimal control problem using KL decomposition and PCE interpolation. This surrogate model may be used for different uncertainty quantification studies as presented in Section 2.5. The proposed approach and the different uncertainty quantification studies are illustrated in the next Section on a two-stage-to-orbit launch vehicle optimal control problem.

\section{Application to launch vehicle optimal control problem}

\subsection{Problem description and considered uncertainties}

In order to illustrate the proposed method to propagate the uncertainties, the optimization of the trajectory of a Two-Stage-To-Orbit launch vehicle is considered. The launch vehicle is composed of two stages with a first stage involving 9 engines and a second stage using only one engine. All the engines use LOx / LCH4 propellant. The launch vehicle has a diameter of $4.6 \mathrm{~m}$ for both stages. The reference mission is relative to the injection of a 7.5 tons payload into a circular $800 \mathrm{~km}$ x $800 \mathrm{~km}$ Sun Synchronous Orbit (SSO). The launch pad is located at the European spaceport (Kourou in French Guiana).

The optimization of the consumption of the launch vehicle is carried out. Here, it is supposed that the architecture of the launch vehicle is already defined and the objective is to minimize the propellant mass needed to inject the considered payload at the given orbit. It is considered that the Gross Lift Off Weight is fixed to 442 tons. 
Table 1

Description of uncertainties

\begin{tabular}{|l|l|l|}
\hline Name & Notation & Model (mean, standard deviation) \\
\hline Specific impulse stage 1 & $U_{I s p_{1}}$ & $\mathcal{N}(0,2)$ (additive, $s$ ) \\
Specific impulse stage 2 & $U_{I s p_{2}}$ & $\mathcal{N}(0,2)$ (additive, $s$ ) \\
Residual mass stage 1 & $U_{m_{1}}$ & $\mathcal{N}(0,1500)$ (additive, $\mathrm{kg}$ ) \\
Residual mass stage 2 & $U_{m_{2}}$ & $\mathcal{N}(0,500)$ (additive, $\mathrm{kg}$ ) \\
Mass flow rate stage 1 & $U_{q_{1}}$ & $\mathcal{N}(0,10)$ (additive, $\mathrm{kg} / \mathrm{s}$ ) \\
Mass flow rate stage 2 & $U_{q_{2}}$ & $\mathcal{N}(0,10)$ (additive, $\mathrm{kg} / \mathrm{s}$ ) \\
Drag coefficient & $U_{C D}$ & $\mathcal{N}(1,0.1)$ (multiplicative, - ) \\
\hline
\end{tabular}

The optimization problem is the following :

$$
\begin{aligned}
\min & \boldsymbol{J}(\mathbf{z}, \mathbf{y}, \mathbf{x}(t), \mathbf{w}(t), \mathbf{U})=\int_{t_{0}}^{t_{f}} \dot{m}(t) d t \\
\text { w.r.t. } & \mathbf{w}(t)=\{\boldsymbol{\theta}, \boldsymbol{\psi}\} \\
\text { s.t. } & \dot{\mathbf{x}}(t)=f(\mathbf{z}, \mathbf{y}, \mathbf{x}(t), \mathbf{w}(t), \mathbf{U}) \\
& \mathbf{g}_{\text {apogee }}(\mathbf{z}, \mathbf{y}, \mathbf{x}(t), \mathbf{w}(t), \mathbf{U}) \leq \epsilon_{\text {apogee }} \\
& \mathbf{g}_{\text {perigee }}(\mathbf{z}, \mathbf{y}, \mathbf{x}(t), \mathbf{w}(t), \mathbf{U}) \leq \epsilon_{\text {perigee }} \\
& \mathbf{g}_{\text {burnt mass }}(\mathbf{z}, \mathbf{y}, \mathbf{x}(t), \mathbf{w}(t), \mathbf{U}) \leq \epsilon_{\text {burnt mass }} \\
& \mathbf{x}_{\text {min }} \leq \mathbf{x}(t) \leq \mathbf{x}_{\max } \quad \forall t \in \mathcal{T} \\
& \mathbf{w}_{\min } \leq \mathbf{w}(t) \leq \mathbf{w}_{\max } \quad \forall t \in \mathcal{T} \\
& \mathbf{x}\left(t_{0}\right)=\mathbf{x}_{0} \\
& \mathbf{x}\left(t_{f}\right)=\mathbf{x}_{f}
\end{aligned}
$$

with $\dot{m}(\cdot)$ the mass flow rate of the launch vehicle, $\theta$ the pitch angle control vector, $\boldsymbol{\psi}$ the heading angle control vector, $\mathbf{g}_{\text {apogee }}(\cdot), \mathbf{g}_{\text {perigee }}(\cdot)$ and $\mathbf{g}_{\text {burnt mass }}(\cdot)$ the constraints about the apogee of the target orbit, the perigee of the target orbit and the available mass of propellant for injecting the payload (and $\epsilon$ the corresponding tolerances). All these variables will be described in the following section. Because the launch vehicle optimization problem presents a large number of multiple optima [7], gradient-based algorithms cannot be used and an evolutionary-based algorithm (Covariance Matrix Adaptation - Evolution Strategy, CMA-ES [22]) is involved in the optimization process [10].

Concerning the uncertainty vector, seven uncertain variables are considered. These variables are summarized in the Table 1 provided by expert knowledge definition. Four modeling uncertainties about the propulsive performance are involved, on the specific impulse $\left(U_{I s p}\right)$ of the two stages and the mass flow rate $\left(U_{q}\right)$. Moreover, to account for structural dry mass uncertainties, penalization on dry mass are considered using $U_{m_{1}}$ and $U_{m_{2}}$. These uncertainties allow to represent the lack of knowledge about the structural masses in the early design phase. Finally, the aerodynamics model that is used also suffers from uncertainties characterized by a multiplicative term on the drag coefficient $U_{C D}$. One can note that all the uncertainty model apart the aerodynamics one are additive.

\subsection{Presentation of the launch vehicle modeling \\ 3.2.1. Multidisciplinary process}

In order to estimate the impact of the considered uncertainties on the trajectory, the performance of the launch vehicle are estimated through a multidisciplinary process composed of the mass and sizing, the aerodynamics, the propulsion and the trajectory. All these different disciplines are integrated into an Multidisciplinary Design Optimization process using an MDA in order to ensure the consistency of the interdisciplinary couplings between the different disciplines [3]. The design process is implemented into the openMDAO framework [20] (Figure 4). The different disciplines are briefly described in this section. A focus is then made on the trajectory discipline in the next section. 


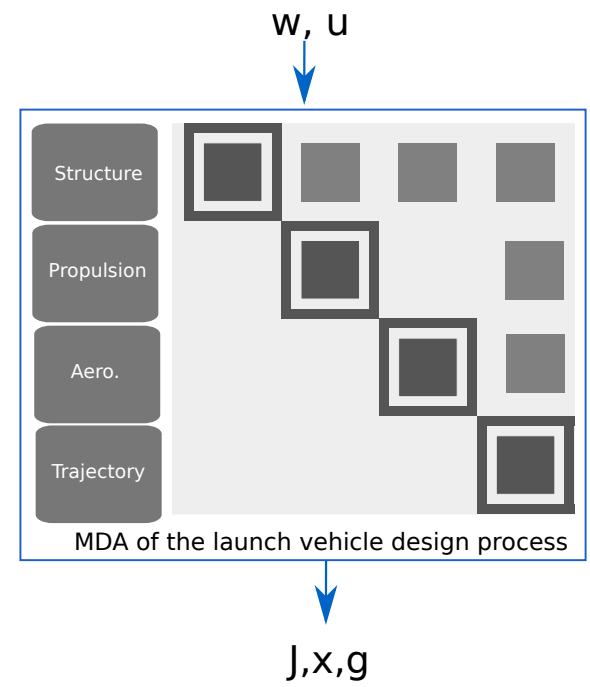

Figure 4: N2 chart of the design process

Propulsion In order to take the propulsive uncertainty into account, a propulsion module has been derived from Rocket Chemical Analysis Equilibrium (CEA) model [25]. This code computes the performance of the rocket engine (specific impulse, thrust) from several inputs (chamber pressure, oxydizer to fuel ratio, etc.). It carries out the thermochemical simulation of complex mixtures, involving combustion of gas in the chamber pressure and expansion in the nozzle. CEA performs theoretical performance of rocket engine calculations that are adapted to conceptual and preliminary design phases. The specific impulse and the mass flow rate computed by CEA are coupling variables that are transmitted to the mass \& sizing and the trajectory disciplines. The nominal value of these variables computed by CEA are then added to the corresponding realization of uncertainties before being transmitted to the other disciplines.

Mass and sizing The mass and sizing module aims at computing the dry mass of the different stages from the propulsion, geometry and trajectory variables. To this end, Mass Estimation Relationships (MERs) have been used from [12] for expandable launch vehicles. All the different components of the launch vehicle (tanks, engine, nozzle, turbopumps, thrust frame, intertank etc.) have been modeled and the masses of these elements are computed using analytical relationships. This module allows to provide a rapid estimation of the dry mass of the launch vehicle depending on the design variables and other disciplines outputs.

Aerodynamics Estimating the aerodynamic performance of this type of vehicles for all the different phases (subsonic, transonic, supersonic and hypersonic) is a quite challenging task. To this end, different engineering tools have been combined by using multi-fidelity model [9]. For the subsonic and transonic flight domains, CFD Euler calculations have been performed using CANOE tool developed at ONERA [13] . For the supersonic and hypersonic flight domains, a combination of results given by a semi-empirical code (MISSILE [14]) and Local Surface Inclination method (SHAMAN) have been used. All the responses of the different codes are aggregated and validated using Gaussian process-based multi-fidelity techniques as detailed in [9]. The drag coefficient given by this module is then combined to the corresponding uncertainty variable and then transmitted to the trajectory module in order to perform the trajectory integration and calculate the performance of the launch vehicle.

\subsubsection{Focus on the trajectory modeling}

The trajectory discipline consists in integrating the system of the ordinary differential equations (equations of motion) according to the time. Here, a single shooting method is used in order to define the optimal control law (parameterized pitch and azimuth angles profiles). The parameters that define the control law $\mathbf{w}(t, \omega)$ are then optimized using a direct single-shooting method [5]. The system of equations of motion $\dot{\mathbf{x}}(t)=f(\mathbf{z}, \mathbf{y}, \mathbf{x}(t), \mathbf{w}(t, \boldsymbol{\omega}), \mathbf{U})$ is defined 
into polar coordinates as follows:

$$
\begin{aligned}
\dot{r} & =v \cdot \sin \gamma \\
\dot{v} & =\frac{T \cos (\theta-\gamma)-D}{m}-g(r) \sin \gamma+\omega_{E}^{2} r \cos \phi(\sin \gamma \cos \phi-\cos \gamma \sin \phi \cos \psi) \\
\dot{\gamma} & =\frac{[L+T \sin (\theta-\gamma)] \cos \mu}{m v}+\left(\frac{v}{r}-\frac{g(r)}{v}\right) \cos \gamma+2 \omega_{E} \sin \psi \cos \phi+\frac{\omega_{E}^{2} r \cos \phi(\cos \gamma \cos \phi+\sin \gamma \sin \phi \cos \psi)}{v} \\
\dot{\lambda} & =\frac{v \cos \gamma \sin \psi}{r \cos \phi} \\
\dot{\phi} & =\frac{v \cos \gamma \cos \psi}{r} \\
\dot{\psi} & =\frac{[L+T \sin (\theta-\gamma)] \sin \mu}{m v \cos \gamma}+\frac{v \cos \gamma \sin \psi \tan \phi}{r}+2 \omega_{E}(\sin \phi-\cos \psi \cos \phi \tan \gamma)+\frac{\omega_{E}^{2} r \sin \phi \cos \phi \sin \psi}{v \cos \gamma} \\
\dot{m} & =-q
\end{aligned}
$$

with :

- $r: \operatorname{radius}(m)$,

- $v$ : norm of the velocity vector $\left(m \cdot s^{-1}\right)$,

- $\gamma$ : flight path angle ( $\mathrm{rad})$,

- $\phi$ : latitude (rad),

- $\lambda$ : longitude (rad),

- $\psi$ : flight path heading ( $\mathrm{rad}$ ),

- $\mu$ : bank angle (rad),

- $\theta$ : pitch angle $(\mathrm{rad})$,

- $\omega_{E}$ : angular velocity of the Earth $(2 \pi / 86164.09 \mathrm{rad} / \mathrm{s})$

- $T$ : Thrust $(N)$

- $D$ : Drag $(N)$,

- $L$ : Lift $(N)$,

- $g(r)$ : gravity acceleration at $r\left(\mathrm{~m}^{2} / \mathrm{s}\right)$,

- $m$ : mass $(k g)$,

- $q$ : mass flow rate $\left(\mathrm{kg} \cdot \mathrm{s}^{-1}\right)$.

The used referential is given in Figure 5. The integration of the system of ordinary differential equations is performed using a $5^{\text {th }}$ order Runge-Kutta method involving the handling of events (fairing jettisoning, change of control law profile as a function of flight conditions, etc.). The control law of the pitch angle is decomposed into different phases [12]: lift-off, pitch-over maneuver, gravity turn and bi-linear tangent law (Figure 6). A discontinuity between gravity turn and bi-linear tangent law is allowed since no more aerodynamics forces are undertaken by the launch vehicle at this altitude. Each phase is parameterized by a set of design variables that are optimized. Concerning the heading angle, the optimal heading angle profile is determined using the target inclination law formula involving the inclination of the target orbit and the local latitude of the vehicle [12]. The engine of the second stage is shut down 


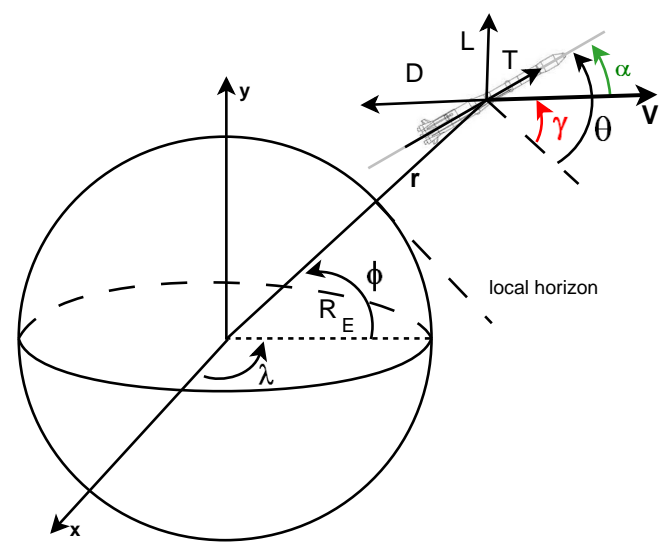

Figure 5: Earth-centered, Earth-fixed reference frame
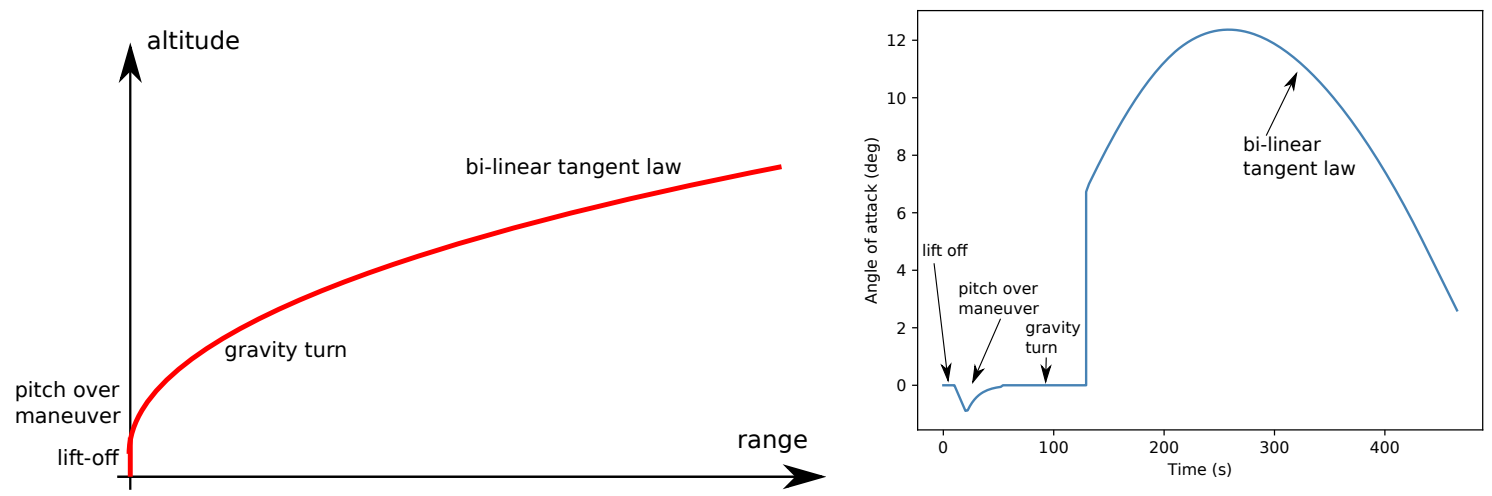

Figure 6: Flight phases of the trajectory (left : altitude as a function of range, right : angle of attack as a function of time)

once the current orbit apogee matches the target apogee. Then, the propellant mass required to circularize the orbit is determined as a function of the final conditions and inclination. Overall, the optimization problem involves eight control variables. Constraints are involved in order to ensure that the reached apogee and perigee match the target ones and the mass of propellant that has been used during the flight is consistent with respect to the rocket architecture. In this paper, an opensource framework (OpenMDAO) is used to integrate trajectory optimization along with the other disciplines (propulsion, structure, aerodynamics). The proposed methodology is non-intrusive and is compatible with the use of alternative trajectory tools.

\subsection{Surrogate model construction and validation}

\subsubsection{Illustration with the altitude state variable}

In this section, the process of surrogate model construction and validation is derived for the launch vehicle test case presented in the previous section. To carry out uncertainty quantification and surrogate modeling, OpenTURNS library is used [4]. Following the proposed approach presented in Section 2, a limited size Monte Carlo Simulation of $M=200$ samples is carried out to generate optimal control solutions and the corresponding state variables. A sensitivity analysis to the Monte Carlo Simulation size is performed in Section 3.3.2. Focusing first on the altitude state variable as a function of time (Figure 7), the centered altitude samples are determined by removing the empirical mean altitude from the samples (right of Figure 7) in order to carry out the KL decomposition.

These state variable samples are then projected using Eq.(2.3) to determine the corresponding value of the random 

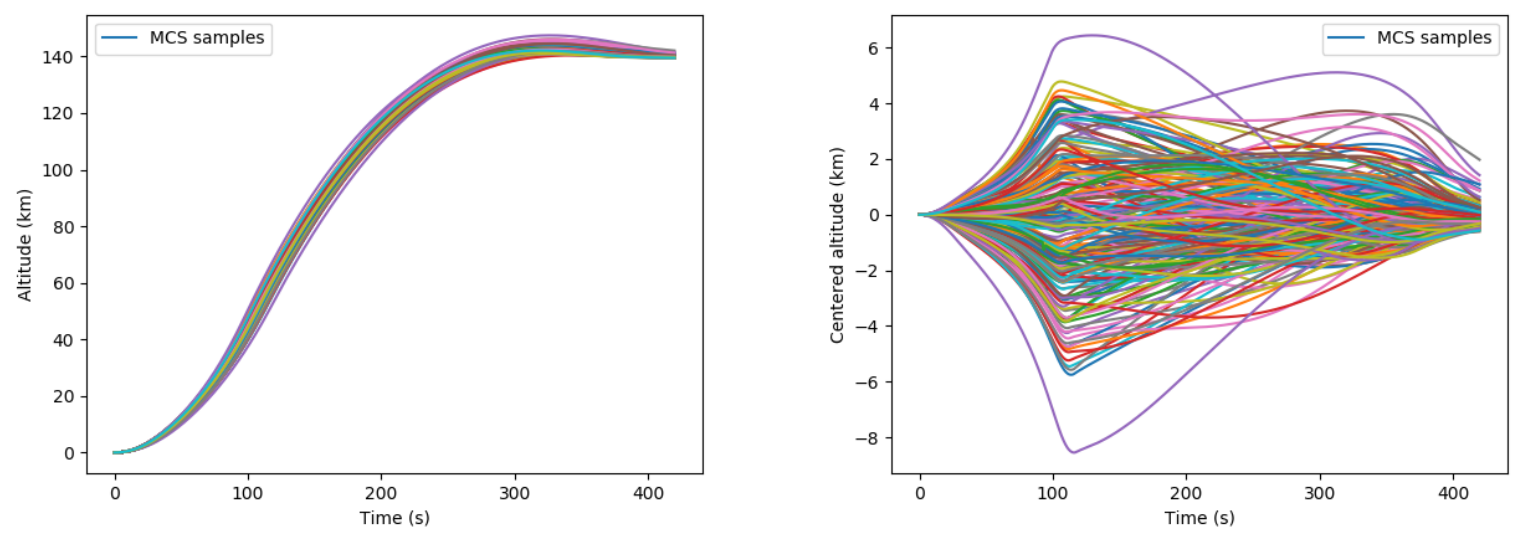

Figure 7: MCS samples altitude as a function of time (left) and centered altitude as a function time (right)

variables into the KL basis for this realization of the state variable. Eventually, the KL expansion prediction is obtained with Eq.(2.4).

To illustrate the influence of the KL truncation, different values of truncation are considered $\epsilon=10^{-2}, 10^{-4}, 10^{-6}$, $10^{-8}, 10^{-10}, 10^{-12}$ corresponding to the value above which the eigenvalues in the KL decomposition are kept. For each KL decomposition corresponding to a different truncation level, the residual samples are determined by computing the difference between the exact obtained validation samples and the predictions based on the KL decomposition using Eq.(2.4). In order to validate the KL decomposition, additional 800 MCS input variable samples are generated and the corresponding optimal state variables are computed by solving the optimal control problem as described in the previous section. These output state variable are used as validation samples $\mathbf{X}_{v}^{*}(t, \mathbf{u})$. The corresponding residual samples are represented in red in Figure 8 along with the centered altitude validation samples. It can be seen in Figure 8, that for $\epsilon=10^{-2}$ (corresponding to only 3 modes in KL decomposition), the residual samples are non negligible. The truncation level of KL is too high. However, by increasing the level of truncation (at $\epsilon=10^{-8}$ for instance), the residual samples become negligible and the KL decomposition is able to predict efficiently the validation samples.

It is also possible to use a model versus surrogate model plot to visualize the quality of the KL decomposition. If the KL decomposition prediction is accurate, in this plot, the data should be aligned along the first bisector. In Figure 9, it can be seen that the KL decomposition for $\epsilon=10^{-2}$ is not accurate enough, but the accuracy improved greatly by increasing the truncation level. The truncation level is a compromise between the accuracy of the KL decomposition and the number of terms in the truncation. Figure 10 illustrates the predictivity factor Q2 as a function of the number of $\mathrm{KL}$ decomposition modes. The predictivity factor $\mathrm{Q} 2$ is defined such that:

$$
Q 2=1-\frac{\sum_{i=1}^{M_{v}}\left(\hat{X}^{*}\left(t, \mathbf{u}_{i}\right)-X_{v}^{*}\left(t, \mathbf{u}_{i}\right)\right)^{2}}{\mathbb{V}\left[X_{v}^{*}(t, \mathbf{u})\right]}
$$

with $M_{v}$ the number of validation samples, $\hat{X}^{*}\left(t, \mathbf{u}_{i}\right)$ the $\mathrm{KL}$ decomposition and $X_{v}^{*}\left(t, \mathbf{u}_{i}\right)$ the exact validation samples. Q2 factor measures the adequacy between the KL decomposition prediction and the exact state variables obtained by optimal control solving.

Table 2 provides the associated Q2 factor and the number of KL decomposition modes. As illustrated with the previous figures, with $\epsilon=10^{-2}$ (corresponding to $3 \mathrm{KL}$ modes), the predictivity factor is not accurate. By increasing the number of KL modes, it converges to 1.0. The level of truncation is a trade-off between the number of nodes (and therefore the number of Polynomial Chaos expansions) and the KL decomposition accuracy. For the altitude variable, a value of $\epsilon=10^{-8}$ is a good compromise between Q2 factor and the number of KL modes.

For the altitude state variable, the first $30 \mathrm{KL}$ decomposition modes (corresponding to $\epsilon=10^{-8}$ ) are represented in Figure 11.

Eventually, based on the KL decomposition (truncated with the first $30 \mathrm{KL}$ modes), Polynomial Chaos Expansions are constructed to relate the input uncertain variables to the state variable prediction. Following the approach proposed 

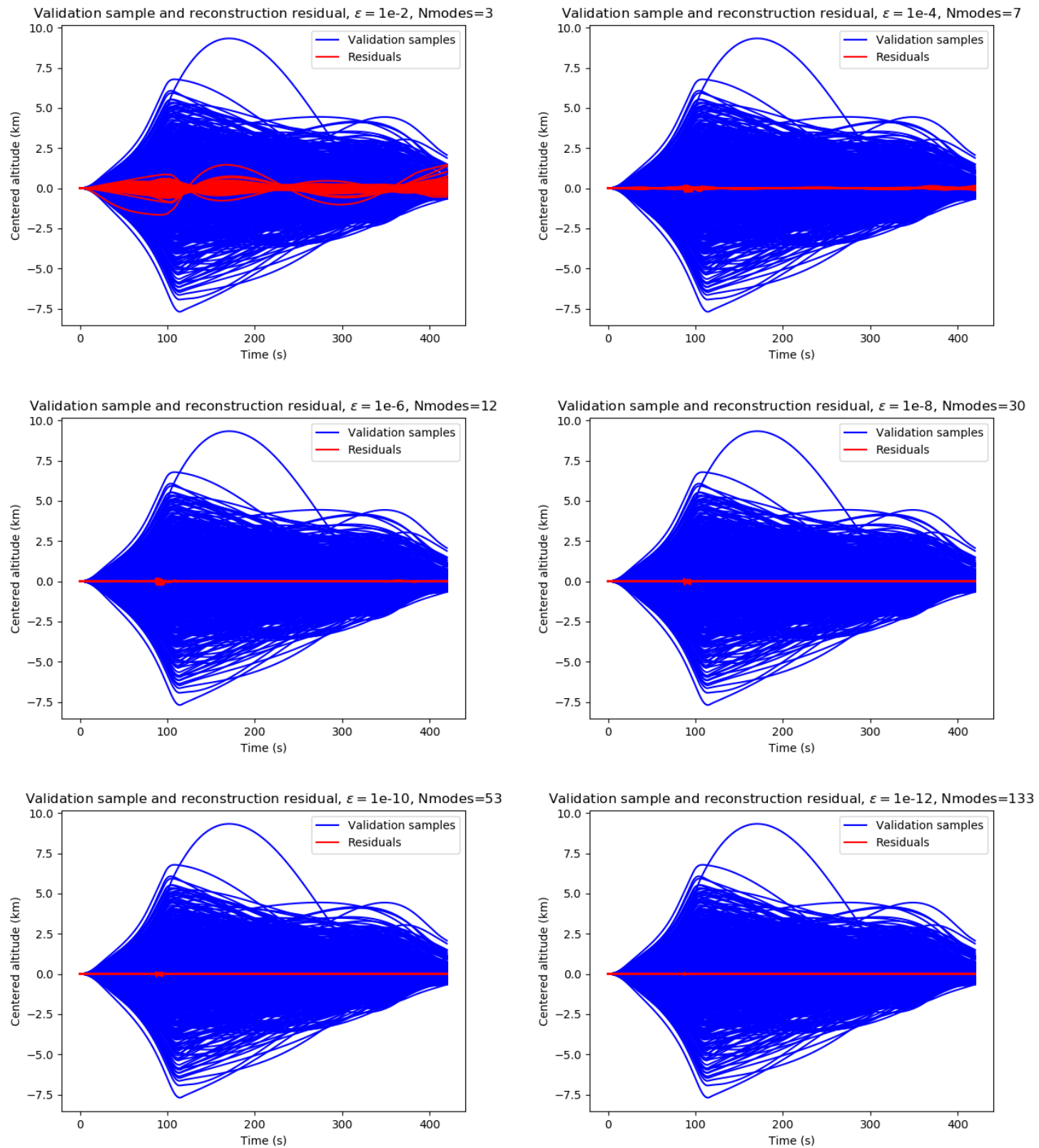

Figure 8: Validation samples and residuals between exact validation samples and $\mathrm{KL}$ prediction

in Section 2.4, for each KL mode, a PCE is determined in order to define the final surrogate model Eq.(30). Then, based on the input variable values generated by MCS for validation, the surrogate model prediction is compared to the exact altitude obtained by solving the optimal control problem (Figure 12).

From Figure 12, the surrogate model predictions and the validation altitudes are similar. It is possible to determine the average Root Mean Square Error (RMSE) along the trajectory (considering all the discretized instants) for the altitude based on the validation set. For the 800 validation samples, the corresponding RMSE of the surrogate model is of $0.564 \mathrm{~km}$, which is limited for a mean trajectory going for $0 \mathrm{~km}$ up to $140 \mathrm{~km}$ with a dispersion of around $10 \mathrm{~km}$ due to input uncertainties. In Figure 13, a comparison of the surrogate model predictions and the validation samples 


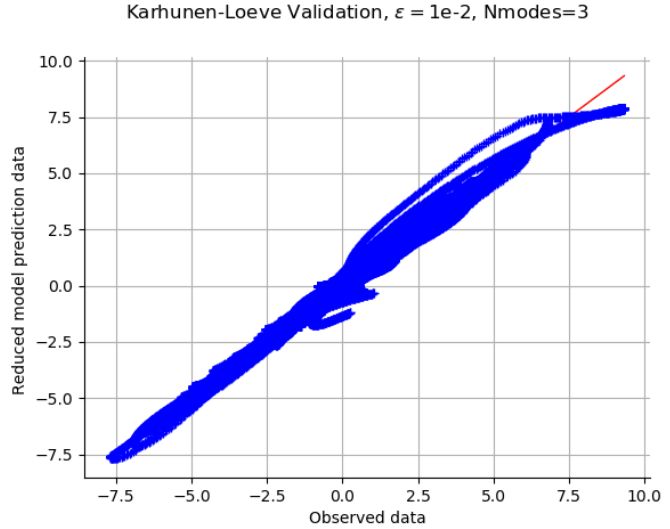

Karhunen-Loeve Validation, $\varepsilon=1 \mathrm{e}-6$, Nmodes $=12$

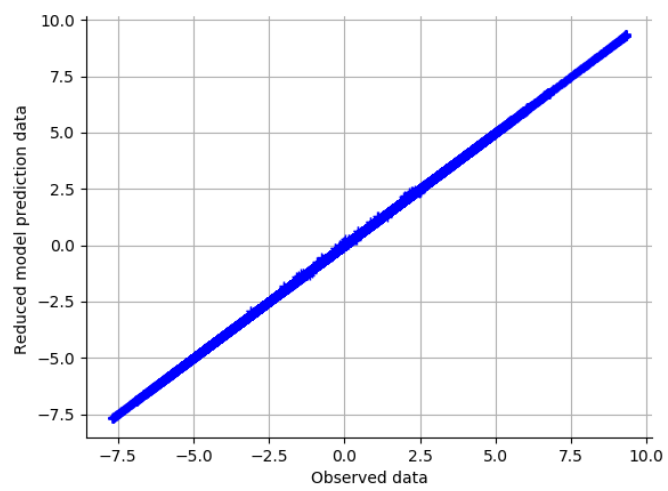

Karhunen-Loeve Validation, $\varepsilon=1 \mathrm{e}-10$, Nmodes $=53$

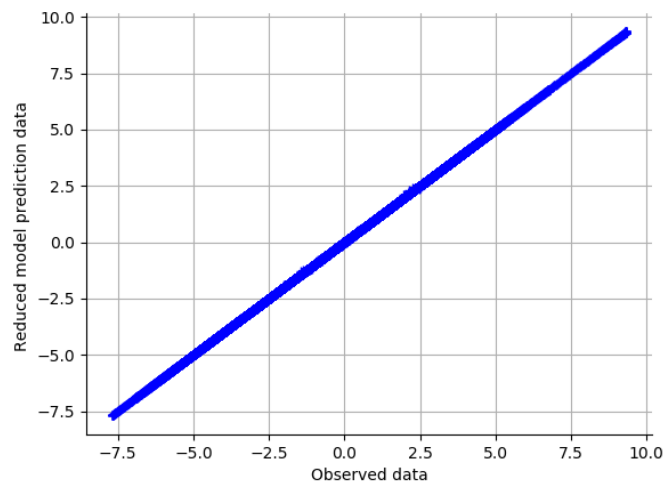

Karhunen-Loeve Validation, $\varepsilon=1 \mathrm{e}-4$, Nmodes $=7$

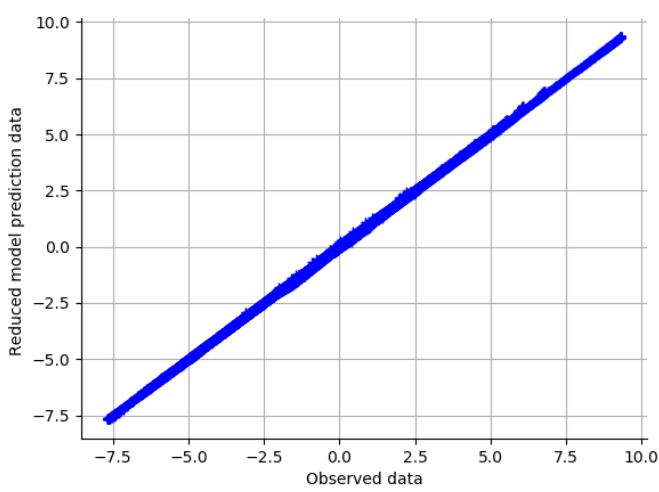

Karhunen-Loeve Validation, $\varepsilon=1 \mathrm{e}-8$, Nmodes $=30$

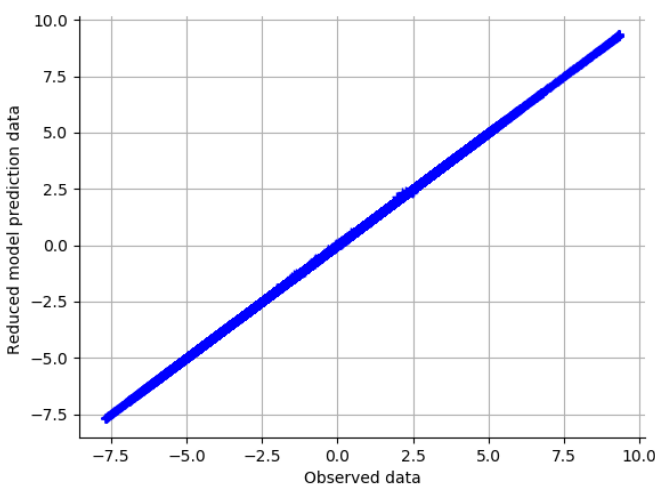

Karhunen-Loeve Validation, $\varepsilon=1 \mathrm{e}-12$, Nmodes $=133$

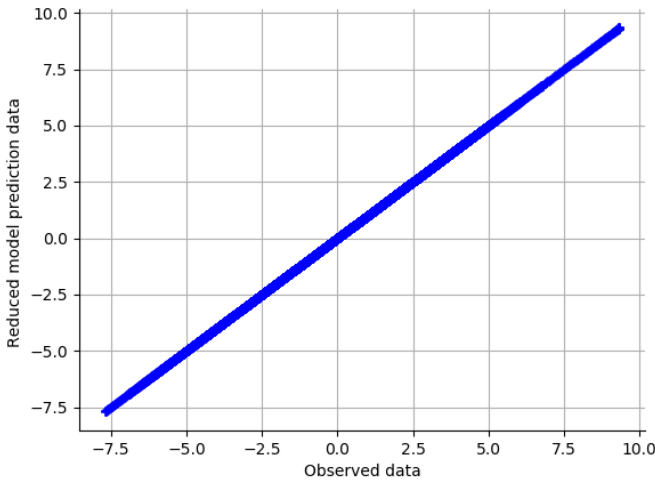

Figure 9: Comparison of validation samples (observed data) and KL prediction samples (reduced model prediction data)

is displayed. The values are aligned along the first bisector (except maybe for one or two realizations). Moreover, the histogram of the error between the prediction and the exact state variable is presented in Figure 13. The error has a mean value of $-0.047 \mathrm{~km}$ and a standard deviation of $0.562 \mathrm{~km}$.

\subsubsection{Sensitivity to Monte Carlo Simulation size}

In order to evaluate the influence of the initial MCS size, the process presented in the previous section is repeated for four sizes of initial MCS samples (50,100, 150 and 200).

The obtained results are presented in Table 3 and Figure 14. The resulting RMSE for the different surrogate models is given in Table 3. As expected, the increase in the number of initial MCS samples (Design Of Experiment - DoE) 

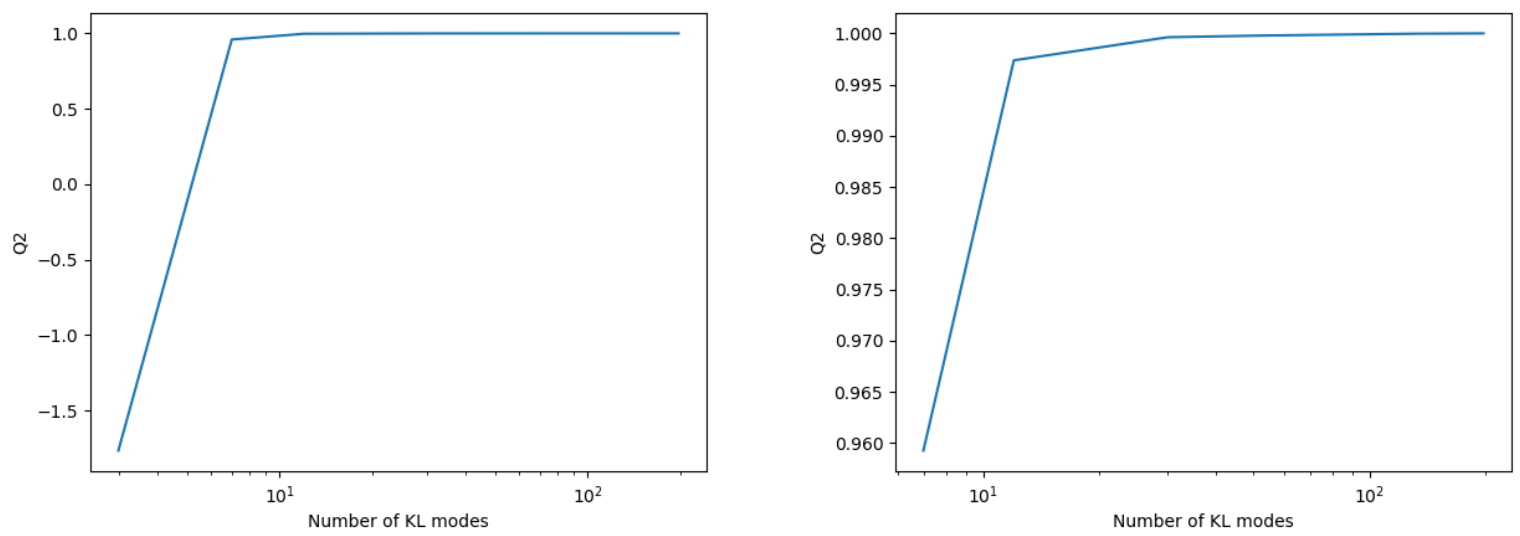

Figure 10: Predictivity factor Q2 as a function of the number of $\mathrm{KL}$ decomposition modes (right, zoom with only a number of $\mathrm{KL}$ modes superior to 7 )

Table 2

Predictivity factor $\mathrm{Q} 2$ as a function of the number of $\mathrm{KL}$ modes

\begin{tabular}{|c|c|c|c|c|c|c|c|}
\hline Number of KL modes & 2 & 7 & 12 & 30 & 53 & 133 & 197 \\
\hline$\epsilon$ & $10^{-2}$ & $10^{-4}$ & $10^{-6}$ & $10^{-8}$ & $10^{-10}$ & $10^{-12}$ & $10^{-14}$ \\
\hline Q2 & -1.76486 & 0.95928 & 0.99736 & 0.99961 & 0.99978 & 0.99996 & 0.99999 \\
\hline
\end{tabular}

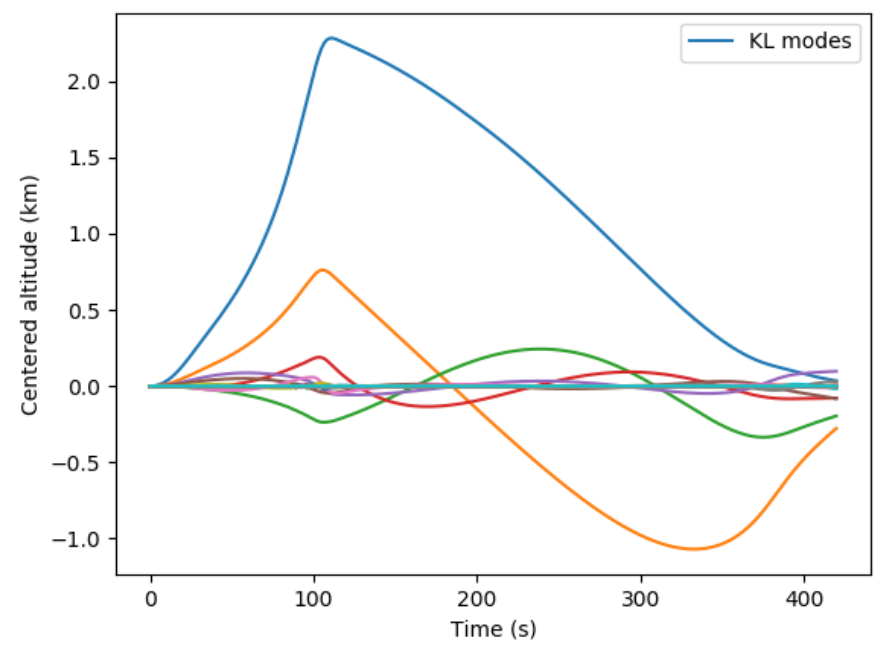

Figure 11: The first $30 \mathrm{KL}$ decomposition modes for the centered altitude

results in a decrease of the RMSE from $1.083 \mathrm{~km}$ with 50 samples up to $0.564 \mathrm{~km}$ for 200 samples. However, the required computational cost (based on parallel calculations on a 6 cores cluster Broadwell Intel Xeon CPU E5-2650 $\mathrm{v} 4$ at $2.20 \mathrm{GHz}$ ) increases consequently. A trade-off between the available time and the accuracy is required. As the acquisition of the state variable data is carried out off-line, in the following, an initial DoE size of 200 samples is considered. To obtained both the 200 samples for the training and the 800 samples for the validation it required $340 \mathrm{~h}$ 


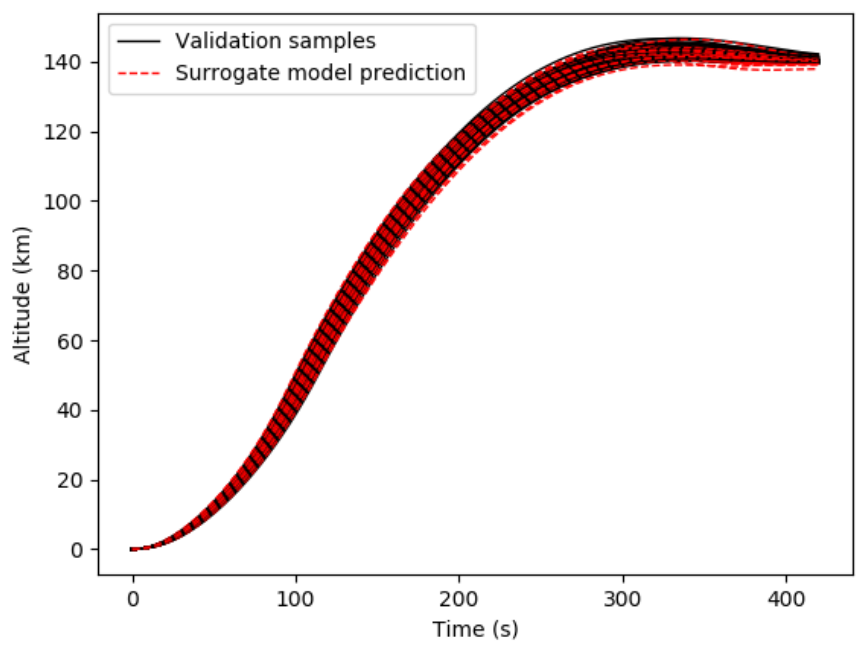

Figure 12: Surrogate model prediction based on 200 MCS samples for the training and $30 \mathrm{KL}$ modes
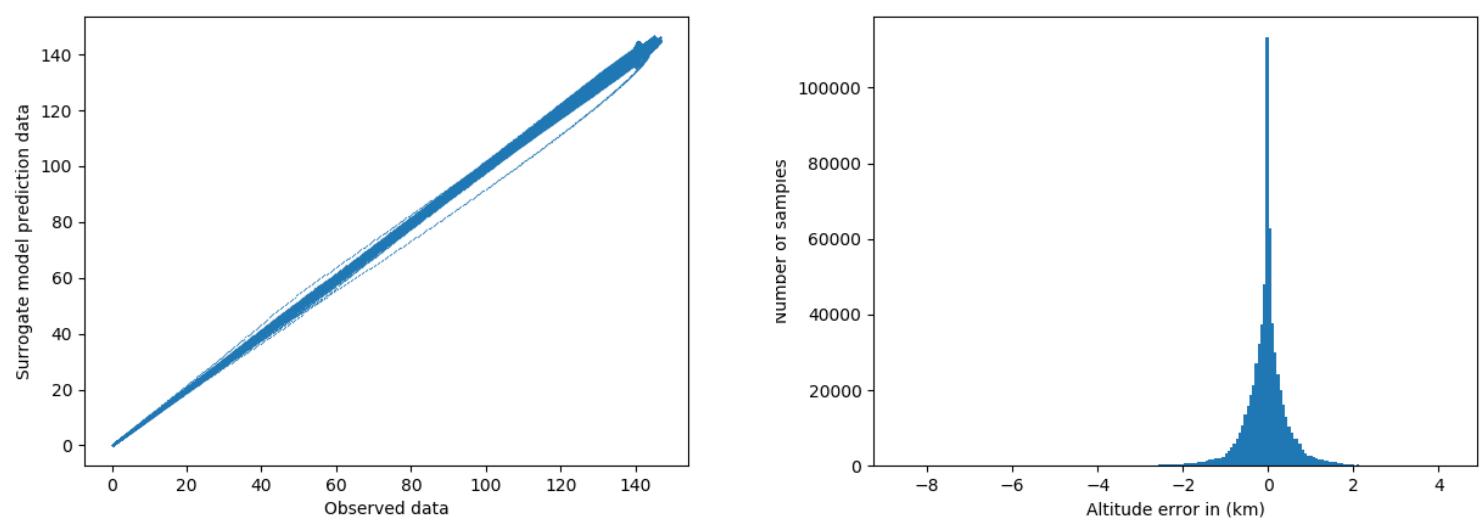

Figure 13: Comparison of validation samples (observed data) and surrogate model prediction (left) and histogram of the prediction error for the validation set for all the discretized instants (right)

\section{Table 3}

Average Root Mean Square Error as a function of the initial MCS size

\begin{tabular}{|c|c|c|c|c|}
\hline Initial MCS size & 50 & 100 & 150 & 200 \\
\hline Computational cost for initial DoE & $17 \mathrm{~h}$ & $34 \mathrm{~h}$ & $51 \mathrm{~h}$ & $68 \mathrm{~h}$ \\
\hline average RMSE $(\mathrm{km})$ & 1.083 & 0.999 & 0.632 & 0.564 \\
\hline
\end{tabular}

(a bit less than 6 days).

\subsubsection{Illustration with other state variables}

Similarly, the proposed approach may be applied to other state variables resulting from the optimal control problem solving such as velocity, mass, flight path angle, etc. but also with quantities of interest derived from the state variable such as the dynamic pressure, the heat flux, the load factor, etc. 

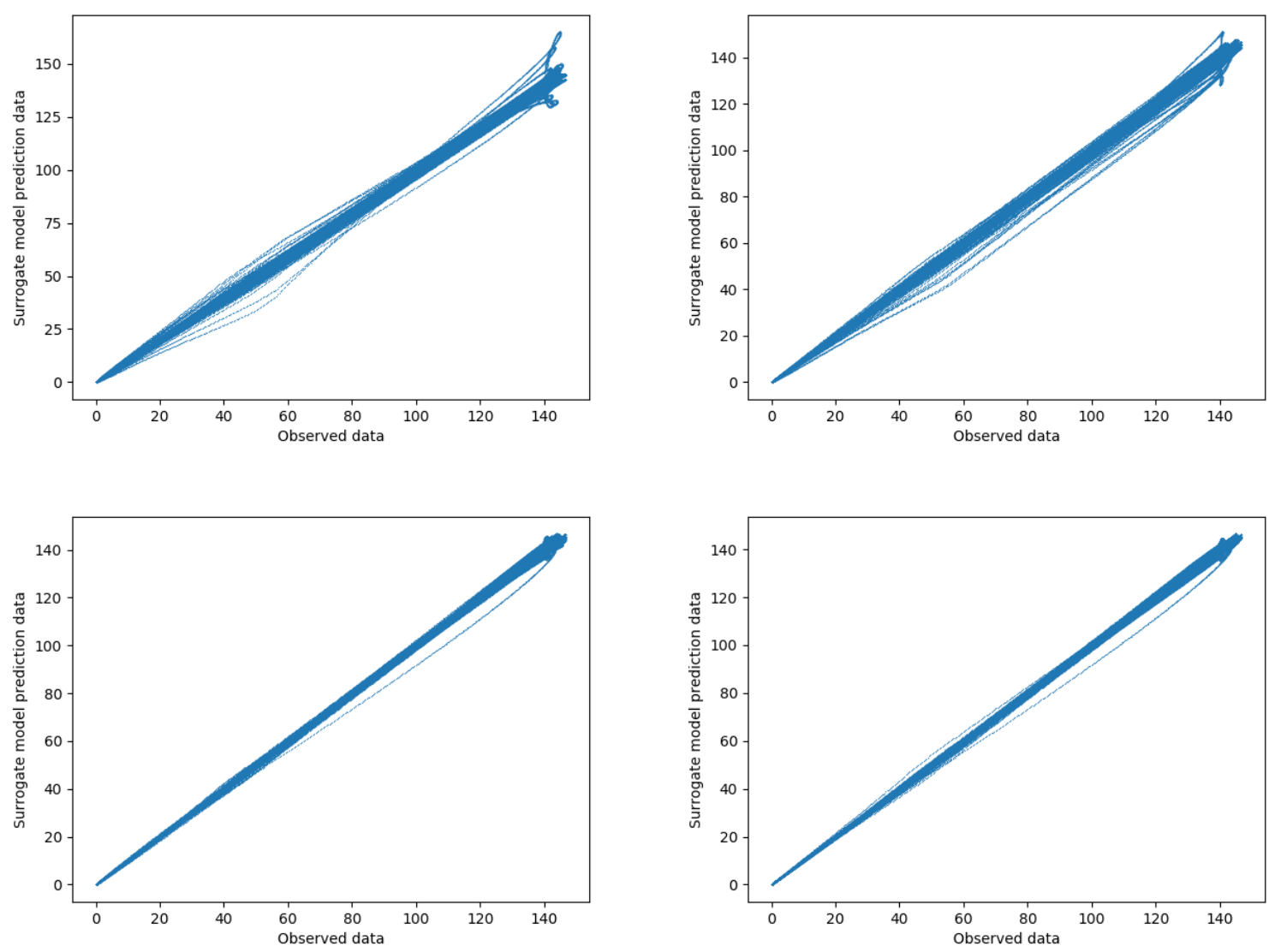

Figure 14: Comparison of validation samples (observed data) and surrogate model prediction for different initial MCS size (50 - upper left, 100 - upper right, 150 - lower left, 200 - lower right)

In Figure 15, the surrogate model predictions for different state variables (velocity, mass, flight path angle) and different quantities of interest (dynamic pressure, heat flux) are represented. For some variables presenting a discontinuity that does not appear at the same instant (for instance the velocity at the stage separation), it is easier to represent the variable as a function of the percent of stage flight (from 0 to 100\%). The proposed approach is able to approximate different types of variable dynamics along time, with linear and nonlinear relations. Therefore, based on a limited size MCS samples, these surrogate models may be used for uncertainty quantification studies for launch vehicle trajectories such as uncertainty propagation, sensitivity analysis and reliability analysis. In the following sections, illustrations of such possible studies with an affordable computational cost are presented.

\subsection{Uncertainty propagation}

Based on the surrogate model constructed for the different state variables and quantities of interest, it is possible to carry out uncertainty propagation to estimate statistical moments of the output at an affordable computational cost. For that, samples from the input variable distributions are drawn and the surrogate model is evaluated (instead of solving the exact optimal control problem) for each input sample. Then, mean value, standard deviation and quantiles may be determined based on the surrogate model responses (or by using PCE coefficient relations for the mean value and the standard deviation).

In this section, the different surrogate models have been trained using 200 MCS samples with a truncation level $\epsilon=10^{-8}$. The estimated values (mean, standard deviation, quantiles) are compared to the values using the validation set composed of 800 MCS samples (by solving the exact optimal control problem), and to the values obtained only by using the training data. It allows to quantify the improvement offered by the surrogate model in terms of prediction. 

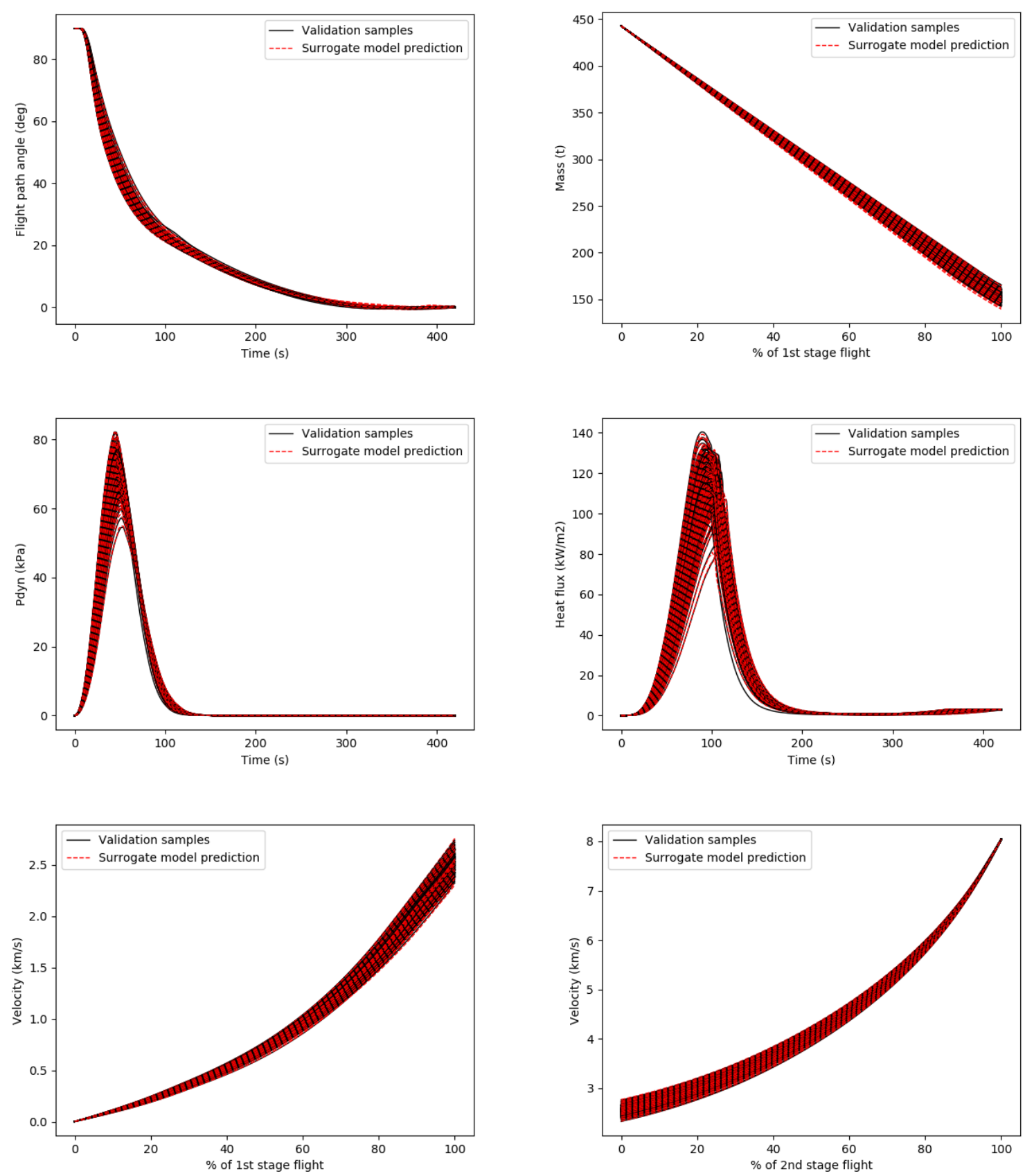

Figure 15: Surrogate model prediction based on 200 MCS samples for the training and 30 KL modes for different state variables and quantities of interest

Considering the state variable velocity, Figure 17 represents the validation set for the velocity (left) and the centered velocity (right - centered velocity).

Using the surrogate model, it is possible to estimate the mean plus the standard deviation for the centered velocity (Figure 18) and to compare it to the value using only the training data (200 samples) and the value using the validation set (800 samples). Using only 200 samples to train the surrogate model and then to use it to predict the mean plus/minus the standard deviation allows to improve the prediction compared to the same estimation using only the 200 




Figure 16: Illustration of generated trajectories
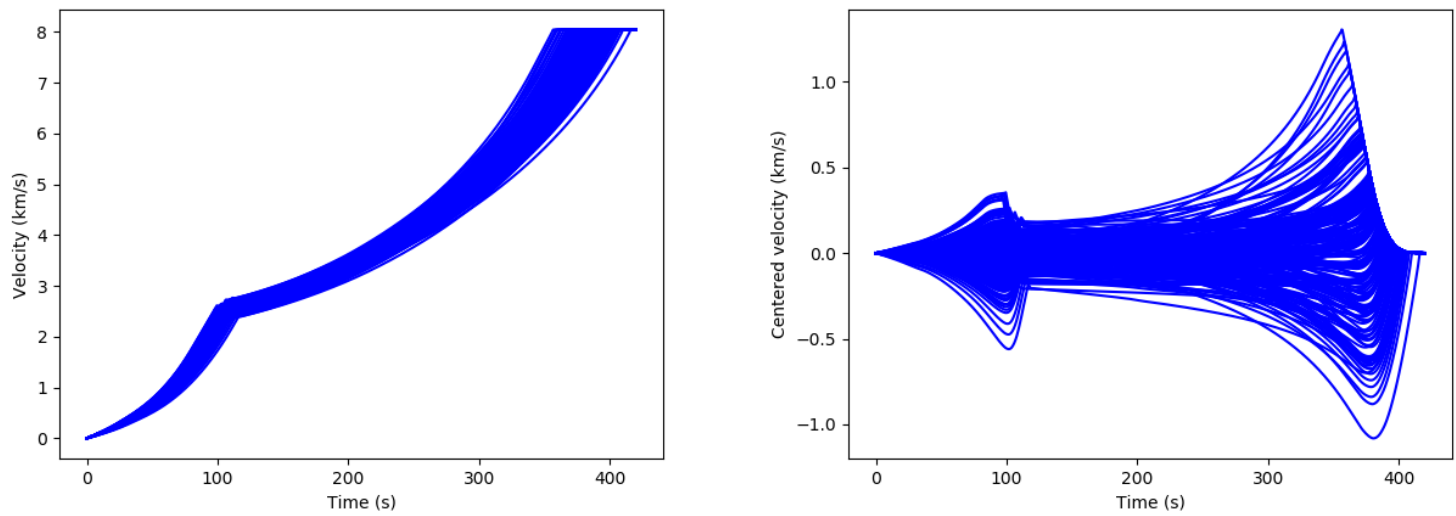

Figure 17: Validation set for the velocity (left) and centered velocity (right) as a function of time

samples. Indeed, in Figure 18, the surrogate model provides almost the same statistical moment results than by using the validation set (800 samples) but only by using 200 samples, reducing the associated computational cost. It can be seen that using alone the 200 samples without a surrogate model is not sufficient to accurately predict the statistical moments of the exact stochastic process.

Similarly, considering the dynamic pressure (Figure 19), it is possible to construct a surrogate model and to use it to estimate quantile values. For instance, in Figure 20, different quantiles are estimated (1\%, 5\%, $95 \%$ and $99 \%)$ using the surrogate model. These values are compared to the estimation of the quantiles using the validation set and using the training set alone. As for the mean and the standard deviation, the surrogate model prediction of the quantiles is almost equal to the one of the validation set but by using 200 MCS samples instead of 800 samples. By comparing the quantiles obtained with the surrogate model and with the training set, it is possible to see the interest of using a surrogate model for uncertainty quantification at an affordable computational cost (Figure 21). 


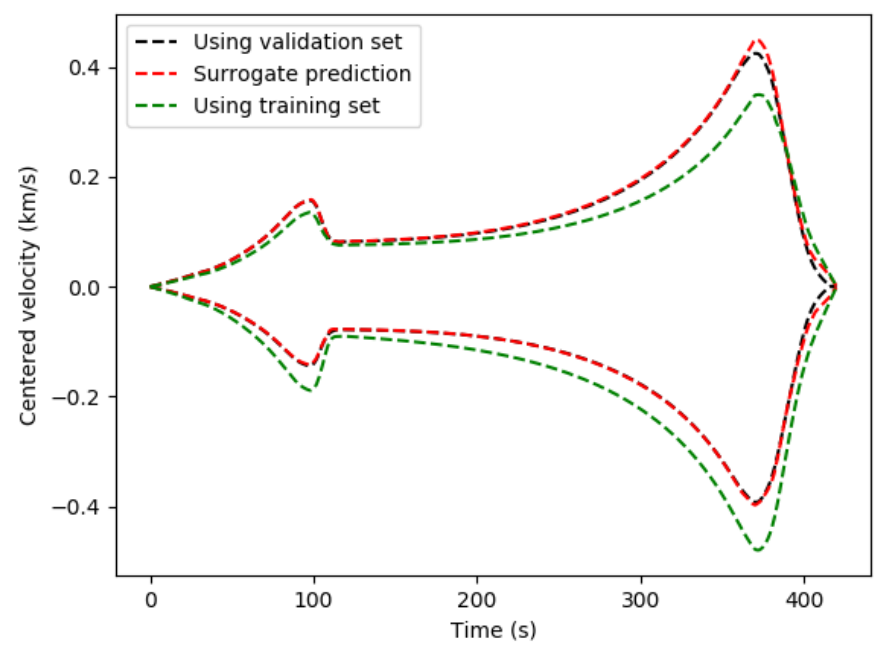

Figure 18: Comparison of the mean plus (above 0) / minus (under 0) standard deviation prediction for the centered velocity
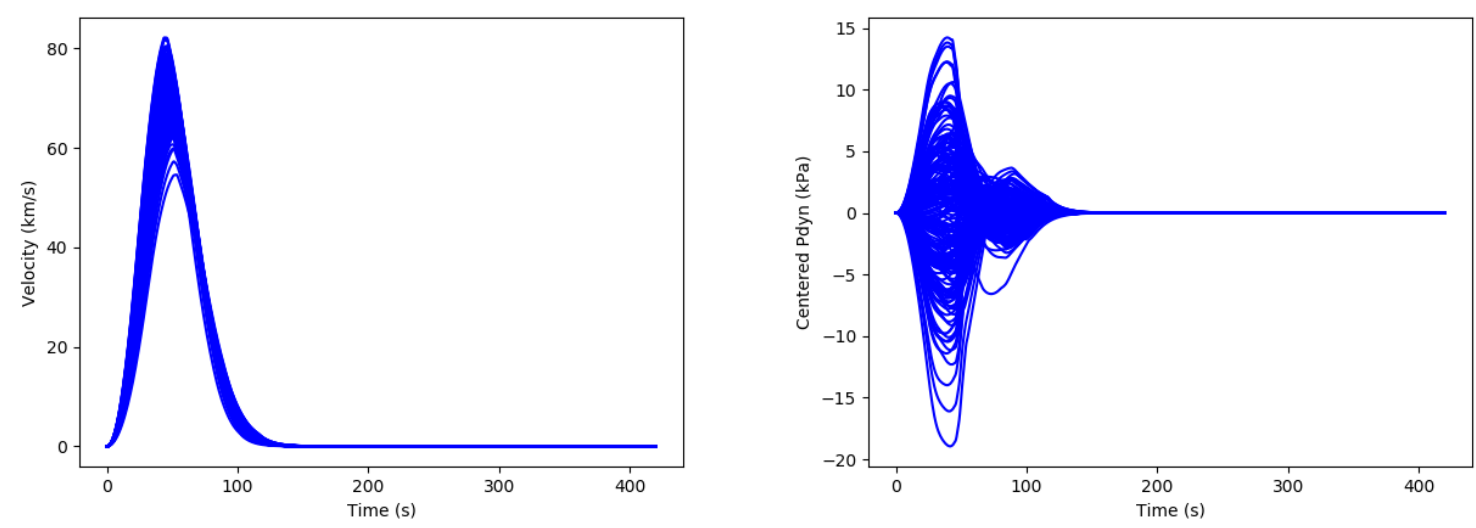

Figure 19: Validation set for the dynamic pressure (left) and centered dynamic pressure (right) as a function of time

\subsection{Reliability analysis}

In order to ensure the safety of space operations, one has to be able to prove that the launch vehicle trajectory will be sufficiently safe with a high level of probability. Among the safety analyses that are needed to ensure that a launch vehicle is reliable (e.g., stage fallback analysis, desorbitation), several ones need to analyze the dynamics of the full trajectory. For example, one can be interested by evaluating the portion of the trajectory that is not visible by the ground stations. Indeed, during the flight, several ground stations are disposed along the trajectory in order to follow the launch vehicle or receive the telemetry information. Examples of ground track for Ariane 5 GTO trajectory is provided in Figure 22. In this section, a simplified problem with three ground stations is considered, defining three cones of visibility (Figure 23).

The goal of this study is to estimate the probability that, due to the modeling uncertainty, the trajectory is not visible by the ground stations for a duration greater than 1 second. The part of the trajectory near the launch pad is not 

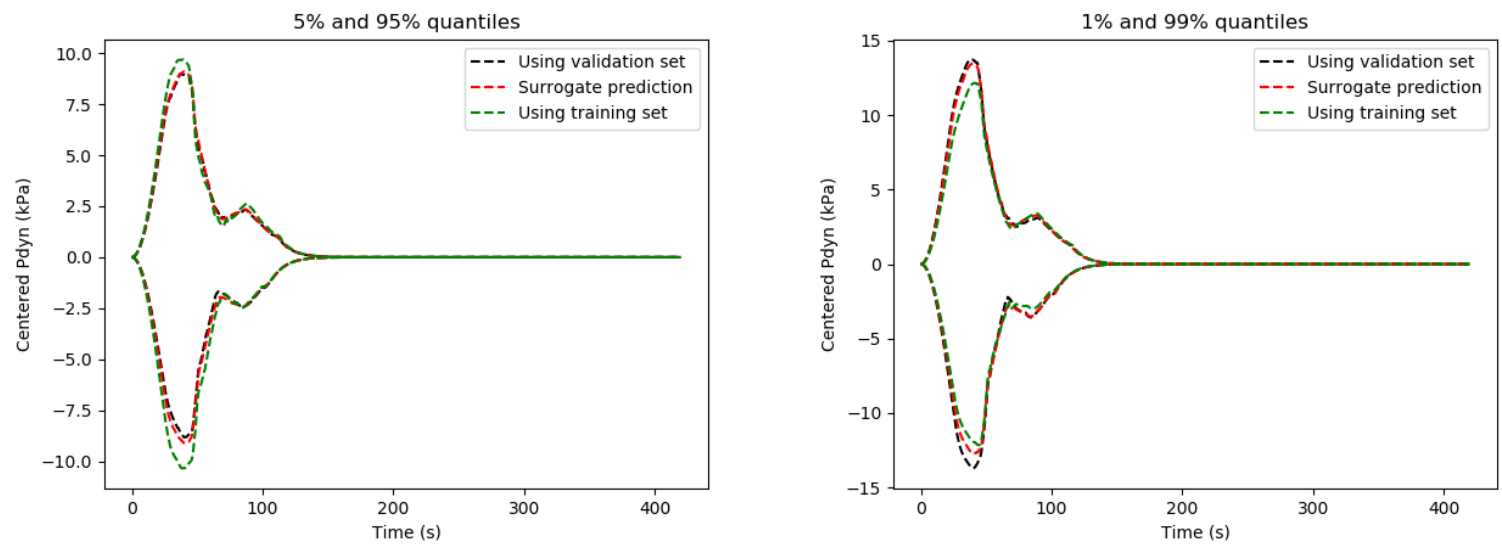

Figure 20: Comparison of the quantiles for the centered dynamic pressure ( $5 \%$ and $95 \%$ - left / $1 \%$ and $99 \%$ - right)

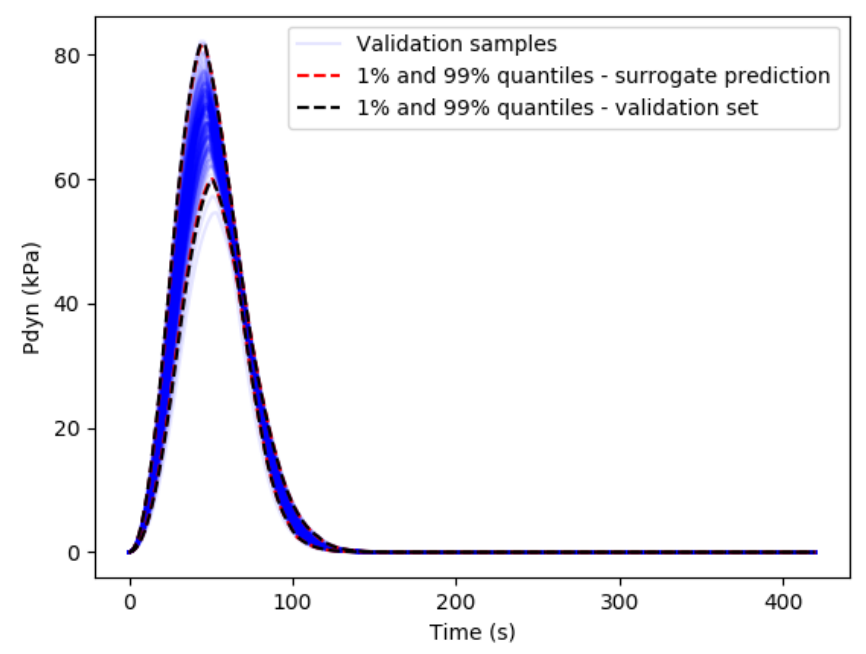

Figure 21: $1 \%$ and $99 \%$ quantiles estimations using the surrogate model and the validation set

considered (altitude under $30 \mathrm{~km}$ ). This probability can be written as follows:

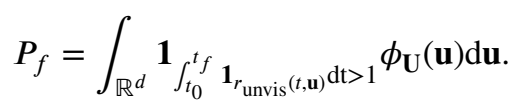

with $r_{\text {unvis }}(t, \mathbf{u})$ a function that takes 1 when the trajectory at the considered instant is not visible by the ground stations and 0 elsewhere. Classical methods to estimate such a probability of failure include Monte-Carlo Simulations [26]. This method consists in generating a set of independent and identically distributed samples of $\mathbf{U}$, performing the trajectory optimization in order to compute the integral of interest by the following formula :

$$
P_{f}^{M C S}=\frac{1}{S} \sum_{i=1}^{S} \mathbf{1}_{\int_{t_{0}}^{t_{f}}} \mathbf{1}_{r_{\text {unvis }}\left(t, \mathbf{u}_{i}\right)} \mathrm{d} \mathrm{t}>1
$$

with $S$ the simulation budget and $\mathbf{u}_{i}, i=1, \ldots, S$ the drawn samples. Unfortunately, for rare event probability estimation, when the simulation budget is limited with regard to magnitude of the probability of interest, this method can be 


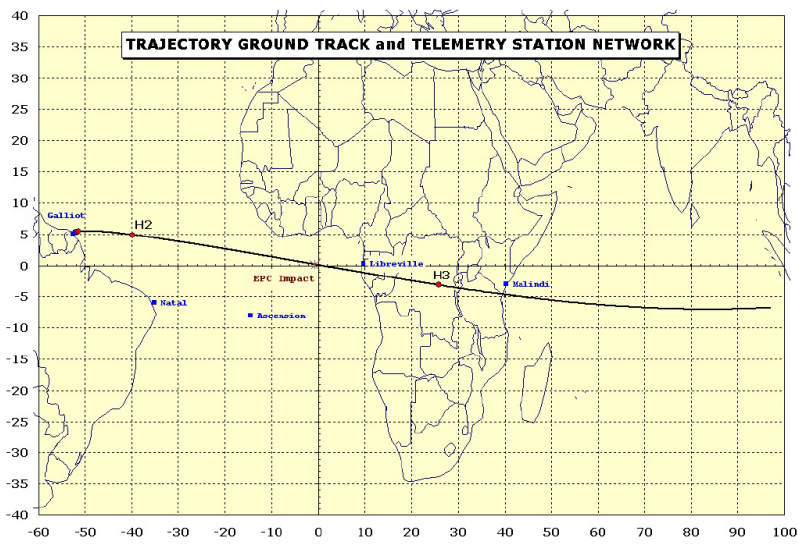

Figure 22: Ground tracking for Ariane 5 GTO trajectory [1]

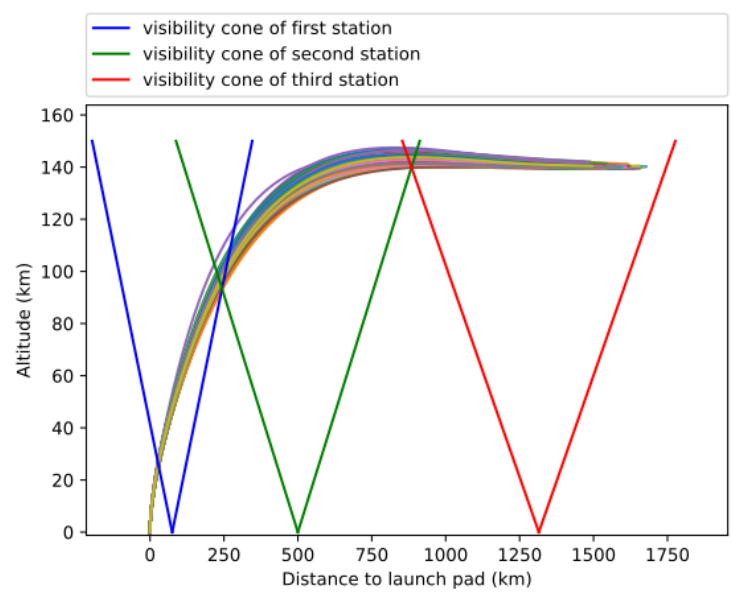

Figure 23: Generated trajectories and visibility cones of ground stations

inaccurate as it can be proven that the relative error of the estimation varies in the following way [27] :

$$
\frac{\sigma_{f}^{P_{f}^{M C S}}}{P_{f}}=\frac{1}{\sqrt{S}} \frac{\sqrt{P_{f}-P_{f}^{2}}}{P_{f}}
$$

Thus, to estimate of probability of $10^{-2}$ with a relative error of $10 \%, 10^{4}$ samples are needed. Thus, as the probability of interest is expected to be very low, it cannot be estimated with using the limited size Monte Carlo Simulations that has been used to learn the surrogate model.

One method to estimate this probability accurately is to generate a large number of samples of uncertain variables and then computing the failure criterion using the surrogate model learnt using the limited size DoE samples (Figure 23). In Table 4, the probability of interest has been estimated using $S=10^{4}$ samples on the learnt model. A parametric analysis with respect to the size of the initial limited size DoE is achieved (Figure 24). As it can be seen, for 200 samples in the DoE, the probability of interest is about $2.7 \times 10^{-2}$ whereas the probability decreases to $1.7 \times 10^{-2}$ for 500 points in the DoE and $8.0 \times 10^{-3}$ for 1000 points. These discrepancies can be partly explained by the fact that the reliability analysis using this kind of model needs to be accurate in the tails of the distribution, where the event of interest (here 


\section{Table 4}

Probability of non visibility for different sizes of the training set (estimated with $10^{5}$ samples)

\begin{tabular}{|c|c|}
\hline Probability of interest & DoE size \\
\hline $2.7 \times 10^{-2}$ & 200 \\
\hline $1.7 \times 10^{-2}$ & 500 \\
\hline $8.0 \times 10^{-3}$ & 1000 \\
\hline
\end{tabular}

the non visibility by ground stations) is located. Unfortunately, few samples relevant to this region are included in the initial DoE. In order to improve the probability estimation, adaptive refinement of the surrogate model needs to be achieved and constitutes an interesting perspective (see discussion in the conclusion). With $10^{5}$ samples, the relative MCS error of this estimation is about $3 \%$ but no information is available on the validity of the surrogate model in the failure regions (compared to the central regions, see discussion in Section 3.3.2) that is why a dedicated refinement strategy is needed.
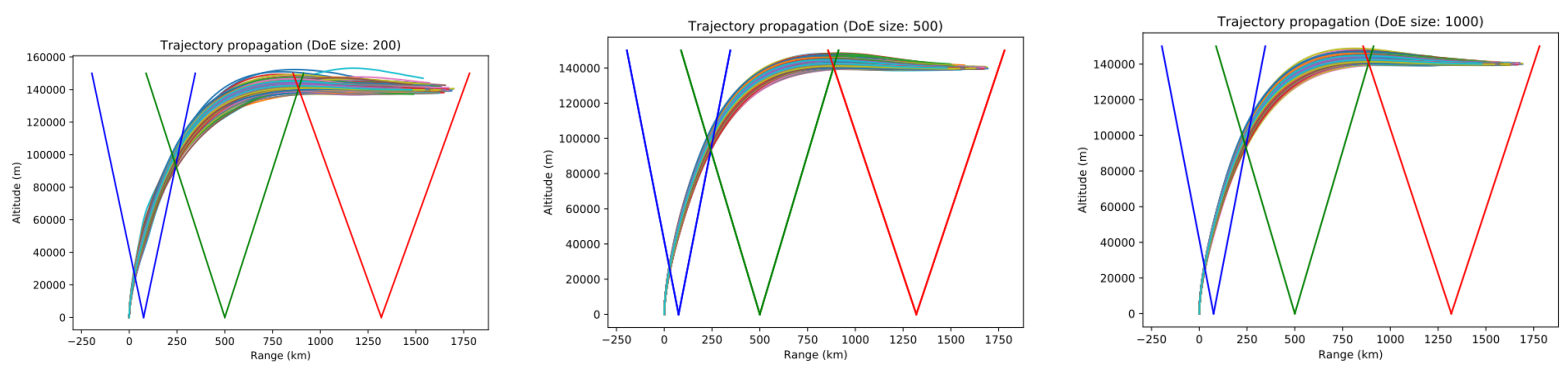

Figure 24: Generated trajectories for different DoE sizes

\subsection{Sensitivity analysis}

Another valuable analysis for understanding the impact of uncertainties in the trajectory can be to carry out time dependent sensitivity analysis of a quantity of interest (e.g., acceleration) with respect to the input uncertain variables. Indeed, such an analysis can provide the designer with precious information to understand which parts of the trajectories are impacted by the uncertainties, in order to prescribe model refinement or trajectory modification to make it less sensitive to the considered uncertainties. These sensitivity analyses can also be used either for screening purpose (fixing the uncertain variables that are the less influential to a nominal value), either for understanding the interaction of the uncertainties that leads to a high variability in the output (e.g., by analyzing high order Sobol'indices).

Following the Sobol' indices derivation in Section 2.5.4, using the 1000 MCS samples to train the proposed surrogate model, it is possible to determine the resulting Sobol' sensitivity indices for the acceleration of the launch vehicle during the accent phase with respect to the input uncertain variables. The acceleration is a valuable state variables in order to perform time related sensitivity analysis because of the direct effect of forces on the acceleration.

Figure 25 presents the first order Sobol' indices as a function of the percent of first stage flight duration for the different input uncertain variables (be careful to the scale color that is different in order to display the differences along the trajectory). The input uncertainty variables $U_{q_{1}}$ (mass flow rate stage 1 ) and $U_{m_{2}}$ (dry mass stage 2 ) are the most influential variables among the different input variables at different instants of the first stage trajectory. It can be seen that $U_{q_{1}}$ is not the most influential variable all along the first stage trajectory, it is highly sensitive in the first $60 \%$ of the first stage trajectory. On the contrary, $U_{m_{2}}$ is mostly influential at the end of the first stage trajectory (above $80 \%$ ). Considering $U_{C D}$ (aerodynamic drag coefficient), it can be seen that it is not an influential variable compared to the other variables however, its highest influence is for instants between $30 \%$ and $50 \%$ of duration of first stage flight which corresponds to the instants at which the launch vehicle crosses the highest density of the atmosphere at a large velocity (highest dynamic pressure), corresponding to altitude between $5 \mathrm{~km}$ and $12 \mathrm{~km}$ in Figure 26.

Similarly for the flight of the second stage, for the Sobol' indices presented in Figure 28, $U_{q_{2}}$ (the mass flow rate of the second stage) and $U_{m_{2}}$ (the dry mass of the second stage) are the most influential variables followed by $U_{I s p_{2}}$ (the 
specific impulse of the second stage). The dry mass of the second stage is mostly influential at the end of the second stage flight duration (above $80 \%$ of flight). As expected, the variables related to the first stage (dry mass, specific impulse, mass flow rate and CD) are the less influential on the second stage acceleration (almost no influence).

Eventually, it is possible to estimate the total order Sobol' indices as explained in Section 2.5.4. These indices give the part of the acceleration variance explained by all the effects in which an input variable plays a part. For instance considering $U_{m_{2}}$, Figure 27 presents the total order Sobol' index as a function of the percent of second stage flight duration. It can be seen that the total order Sobol' index is higher than the first order Sobol index for $U_{m_{2}}$, therefore it indicates that the variable interacts with other variables to contribute to the acceleration variance. 



Figure 25: First order Sobol' indices as a function of the $\%$ of first stage flight for the different input uncertain variables Loic Brevault, Mathieu Balesdent: Preprint submitted to Elsevier 


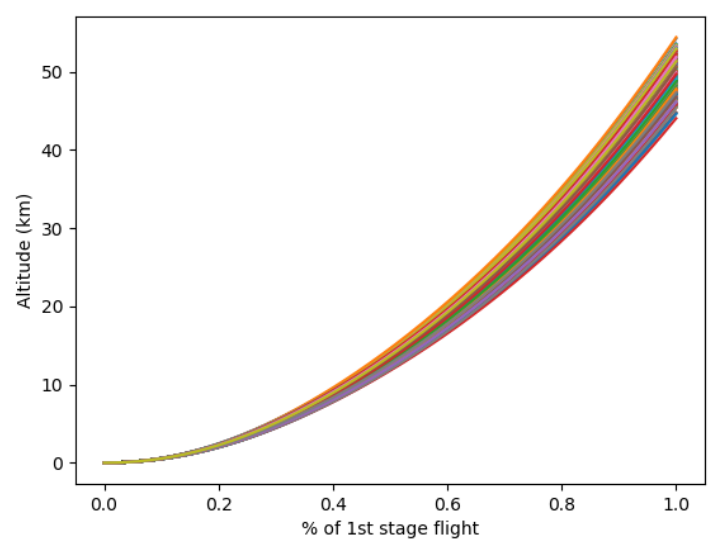

Figure 26: Altitude of launch vehicle as a function of percent of duration of first stage flight

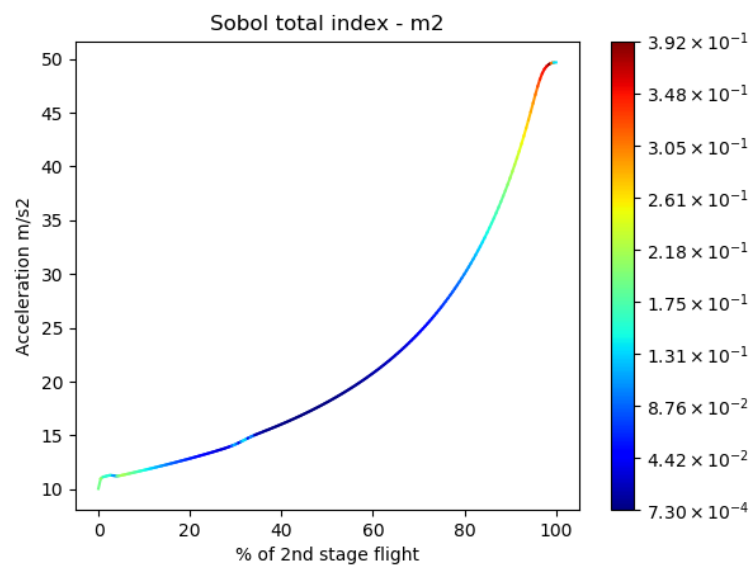

Figure 27: Total order Sobol' index for $U_{m_{2}}$ as a function of the percent of second stage flight duration 

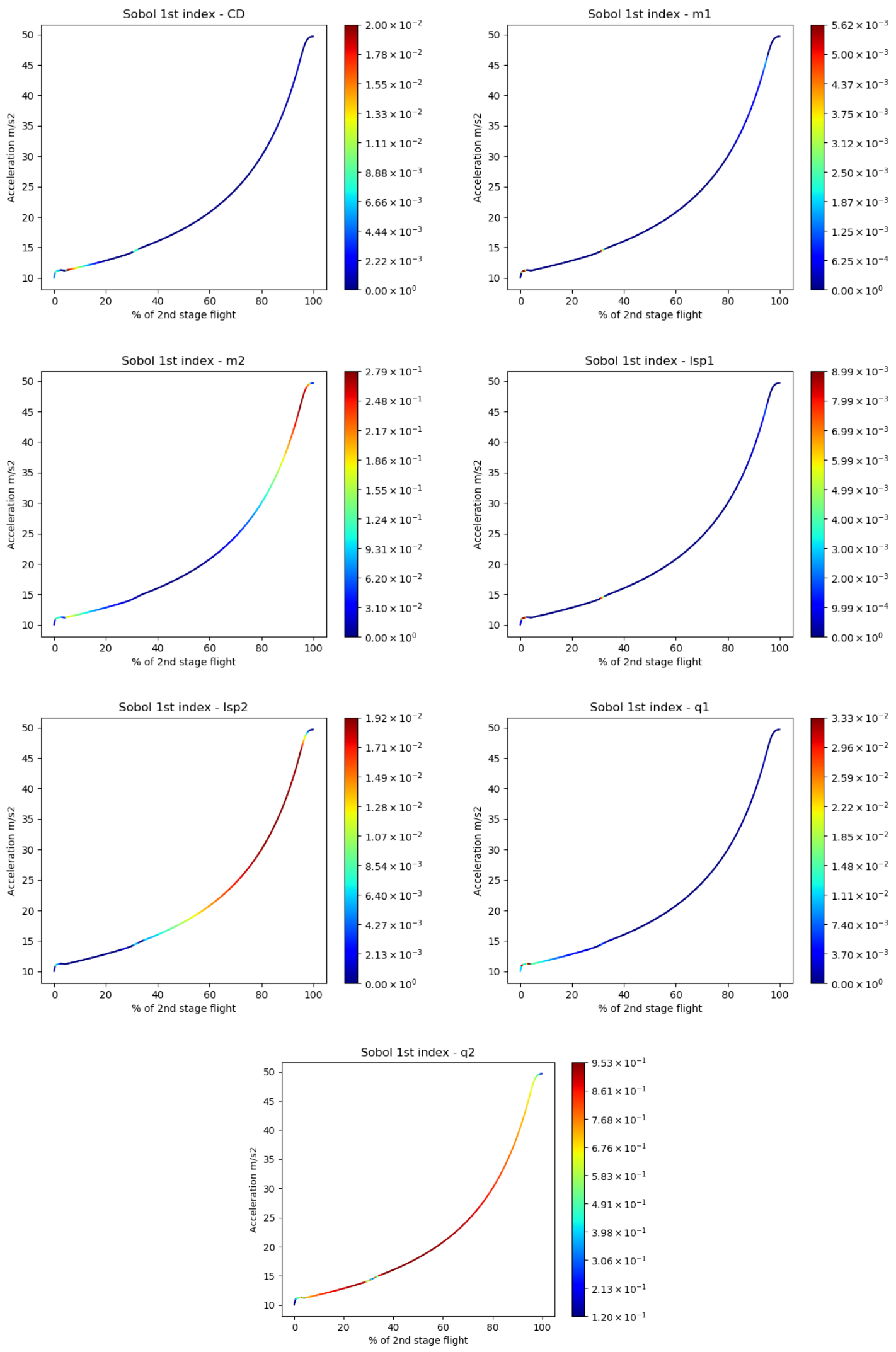

Figure 28: First order Sobol' indices as a function of the $\%$ of second stage flight for the different input uncertain variables Loic Brevault, Mathieu Balesdent: Preprint submitted to Elsevier

Page 31 of 33 


\section{Conclusions}

In this paper, a methodology to propagate uncertainties for launch vehicle trajectory optimization based on a surrogate model technique has been presented in order to tackle the numerical cost of uncertainty quantification studies for coupled multidisciplinary problems involving trajectory optimization. The proposed four steps approach starts with a limited size Monte Carlo simulation (MCS) with the solving of the exact optimal control problem for the MCS samples. Then, a Karhunen-Loève (KL) decomposition is carried out to decompose the state variable stochastic processes. Then, using Polynomial Chaos Expansion of the random variables involved in the KL expansion, it is possible to define a surrogate model that relates the input uncertain variables to the optimal state variables under uncertainty of the trajectory. This surrogate model may be used in a final step for uncertainty quantification studies. The proposed methodology has been applied on a two-stage-to-orbit launch vehicle test case. A discussion on the accuracy of the KL decomposition and the obtained surrogate model as a function of the number of initial Monte Carlo samples highlighted the trade-off between the surrogate model accuracy and the associated computational cost. Then, uncertainty quantification studies have been carried out to estimate state variable statistical moments, quantiles to determine flight envelops, reliability analysis to ensure the visibility of the launcher along its trajectory and eventually sensitivity analysis to estimate the influence of the input uncertain variables on the acceleration of the launch vehicle all along its trajectory.

In terms of future works, in order to ensure the accuracy of the surrogate model especially for reliability analysis, an active learning strategy could be proposed to refine the surrogate model near the failure regions with the highest probability content. It would allow to improve the surrogate model accuracy by adding specific realizations of the stochastic process determined by a refinement criterion. Moreover, the proposed surrogate model approach could be extended to account for design variables in order to solve Uncertainty-based Multidisciplinary Design Optimization problem for launch vehicle design.

\section{Funding Sources}

This work is part of the HERACLES project funded by ONERA - The French Aerospace Lab.

\section{Acknowledgments}

The authors would like to thank Sylvain Dubreuil (ONERA) for the fruitful discussions around Karhunen-Loève, Polynomial Chaos Expansion and Sobol' indices.

\section{References}

[1] Arianespace. “Ariane 5 user's manual”. In: Issue 5, revision 0 (2008).

[2] Richard Askey and James Arthur Wilson. Some basic hypergeometric orthogonal polynomials that generalize Jacobi polynomials. Vol. 319. American Mathematical Soc., 1985.

[3] Mathieu Balesdent et al. "A survey of multidisciplinary design optimization methods in launch vehicle design". In: Structural and Multidisciplinary optimization 45.5 (2012), pp. 619-642.

[4] Michaël Baudin et al. “OpenTURNS: An industrial software for uncertainty quantification in simulation”. In: arXiv preprint arXiv:1501.05242 (2015).

[5] John T Betts. "Survey of numerical methods for trajectory optimization". In: Journal of guidance, control, and dynamics 21.2 (1998), pp. 193-207.

[6] Joseph-Frédéric Bonnans et al. Numerical optimization: theoretical and practical aspects. Springer Science \& Business Media, 2006.

[7] Loic Brevault, Mathieu Balesdent, and Ali Hebbal. "Multi-objective multidisciplinary design optimization approach for partially reusable launch vehicle design”. In: Journal of Spacecraft and Rockets 57.2 (2020), pp. 373-390.

[8] Lo1c Brevault and Mathieu Balesdent. "Uncertainty-Based Multidisciplinary Design Optimization (UMDO)". In: Aerospace System Analysis and Optimization in Uncertainty. Springer, 2020, pp. 235-292.

[9] Loic Brevault, Mathieu Balesdent, and Ali Hebbal. "Overview of Gaussian process based multi-fidelity techniques with variable relationship between fidelities, application to aerospace systems”. In: Aerospace Science and Technology 107 (2020), p. 106339.

[10] Loic Brevault, Mathieu Balesdent, Jérôme Morio, et al. Aerospace System Analysis and Optimization in Uncertainty. Springer, 2020.

[11] Loic Brevault et al. "Decoupled multidisciplinary design optimization formulation for interdisciplinary coupling satisfaction under uncertainty”. In: AIAA Journal 54.1 (2016), pp. 186-205. 
Uncertainty quantification for launch vehicle multidisciplinary design using model order reduction and spectral methods

[12] Francesco Castellini. "Multidisciplinary design optimization for expendable launch vehicles". PhD thesis. Politecnico di Milano, 2012.

[13] Sebastien Defoort et al. "Conceptual design of disruptive aircraft configurations based on High-Fidelity OAD process". In: 2018 Aviation Technology, Integration, and Operations Conference. 2018, p. 3663.

[14] Pascal Denis. “ONERA's aerodynamic prediction code- MISSILE”. In: RTO/AGARD, Symposium on Missile Aerodynamics, Sorrento, Italy, May 11-14, 1998, ONERA, TP. 1998-56. 1998.

[15] Michael Eldred. "Recent advances in non-intrusive polynomial chaos and stochastic collocation methods for uncertainty analysis and design". In: 50th AIAA/ASME/ASCE/AHS/ASC Structures, Structural Dynamics, and Materials Conference 17th AIAA/ASME/AHS Adaptive Structures Conference 11th AIAA No. 2009, p. 2274.

[16] James Fisher and Raktim Bhattacharya. "Optimal trajectory generation with probabilistic system uncertainty using polynomial chaos". In: Journal of dynamic systems, measurement, and control 133.1 (2011).

[17] Roger G Ghanem and Pol D Spanos. "Spectral stochastic finite-element formulation for reliability analysis". In: Journal of Engineering Mechanics 117.10 (1991), pp. 2351-2372.

[18] Anupriya Gogna and Akash Tayal. "Metaheuristics: review and application". In: Journal of Experimental \& Theoretical Artificial Intelligence 25.4 (2013), pp. 503-526.

[19] L Le Gratiet, Stefano Marelli, and Bruno Sudret. "Metamodel-based sensitivity analysis: polynomial chaos expansions and Gaussian processes". In: arXiv preprint arXiv:1606.04273 (2016).

[20] Justin S Gray et al. "OpenMDAO: An open-source framework for multidisciplinary design, analysis, and optimization”. In: Structural and Multidisciplinary Optimization 59.4 (2019), pp. 1075-1104.

[21] Nathan Halko et al. "An algorithm for the principal component analysis of large data sets". In: SIAM Journal on Scientific computing 33.5 (2011), pp. 2580-2594.

[22] Nikolaus Hansen, Sibylle D Müller, and Petros Koumoutsakos. "Reducing the time complexity of the derandomized evolution strategy with covariance matrix adaptation (CMA-ES)". In: Evolutionary computation 11.1 (2003), pp. 1-18.

[23] Michiel JW Jansen. “Analysis of variance designs for model output”. In: Computer Physics Communications 117.1-2 (1999), pp. 35-43.

[24] Xiang Li et al. "Aircraft robust trajectory optimization using nonintrusive polynomial chaos". In: Journal of Aircraft 51.5 (2014), pp. 15921603.

[25] Bonnie J McBride. Computer program for calculating and fitting thermodynamic functions. Vol. 1271. National Aeronautics and Space Administration, Office of Management, 1992.

[26] Nicholas Metropolis and Stanislaw Ulam. "The Monte Carlo method". In: Journal of the American statistical association 44.247 (1949), pp. 335-341.

[27] Jérôme Morio and Mathieu Balesdent. Estimation of rare event probabilities in complex aerospace and other systems: a practical approach. Woodhead Publishing, 2015.

[28] Anil V Rao. “A survey of numerical methods for optimal control”. In: Advances in the Astronautical Sciences 135.1 (2009), pp. 497-528.

[29] Andrea Saltelli, Stefano Tarantola, and KP-S Chan. "A quantitative model-independent method for global sensitivity analysis of model output”. In: Technometrics 41.1 (1999), pp. 39-56.

[30] Ilya Sobol. "On sensitivity estimation for nonlinear mathematical models". In: Matematicheskoe modelirovanie 2.1 (1990), pp. 112-118.

[31] Ilya M Sobol. "Global sensitivity indices for nonlinear mathematical models and their Monte Carlo estimates". In: Mathematics and computers in simulation 55.1-3 (2001), pp. 271-280.

[32] Ilya M Sobol. "Sensitivity analysis for non-linear mathematical models". In: Mathematical modelling and computational experiment 1 (1993), pp. 407-414.

[33] Christian Soize and Roger Ghanem. "Physical systems with random uncertainties: chaos representations with arbitrary probability measure". In: SIAM Journal on Scientific Computing 26.2 (2004), pp. 395-410.

[34] Bruno Sudret. "Global sensitivity analysis using polynomial chaos expansions". In: Reliability engineering \& system safety 93.7 (2008), pp. 964-979.

[35] Bruno Sudret and Armen Der Kiureghian. Stochastic finite element methods and reliability: a state-of-the-art report. Department of Civil and Environmental Engineering, University of California, 2000.

[36] Fenggang Wang et al. "Robust trajectory optimization using polynomial chaos and convex optimization". In: Aerospace Science and Technology 92 (2019), pp. 314-325.

[37] Norbert Wiener. “The homogeneous chaos”. In: American Journal of Mathematics 60.4 (1938), pp. 897-936.

[38] Fenfen Xiong, Ying Xiong, and Bin Xue. "Trajectory optimization under uncertainty based on polynomial chaos expansion". In: AIAA guidance, navigation, and control conference. 2015, p. 1761.

[39] Reza Zardashti et al. "Robust Optimum Trajectory Design of a Satellite Launch Vehicle in the Presence of Uncertainties". In: Journal of Aerospace Technology and Management 12 (2020). 